\title{
Estimating the joint statistics of images using Non-Parametric Windows with application to registration using Mutual Information
}

\author{
Nicholas Dowson Member, IEEE, Timor Kadir and Richard Bowden Senior Member, IEEE
}

\begin{abstract}
Recently, Non-Parametric (NP) Windows has been proposed to estimate the statistics of real $1 \mathrm{D}$ and $2 \mathrm{D}$ signals. NP Windows is accurate, because it is equivalent to sampling images at a high (infinite) resolution for an assumed interpolation model. This paper extends the proposed approach to consider joint distributions of image-pairs. Secondly, Green's Theorem is used to simplify the previous NP Windows algorithm. Finally, a resolution aware NP Windows algorithm is proposed, to improve robustness to relative scaling between an image-pair. Comparative testing of $2 \mathrm{D}$ image registration was performed using translation only and affine transformations. Although more expensive than other methods, NP Windows frequently demonstrated superior performance for bias (distance between ground truth and global maximum) and frequency of convergence. Unlike other methods, the number of samples and number of bins has little effect on NP Windows, and the prior selection of a kernel is not required.
\end{abstract}

Index Terms-Mutual Information, Joint image statistics, Registration, Sampling.

\section{INTRODUCTION}

The estimation of accurate image statistics is important for many computer vision applications. For example, joint statistics estimation underlies the alignment or registration of pairs of images through maximisation of their Mutual Information (MI); here MI is typically estimated from the joint-intensity histogram of the image pair. Image and template matching is the particular application of interest in this paper.

MI has been widely used in registration applications since its concurrent introduction by Viola \& Wells [1], Collignon et al. [2] and Studholme et al. [3], to the image processing community. Its popularity stems from the robustness of MI to occlusion and noise, and its tolerance of non-linear intensity relationships [4]. The latter trait is useful for registering multimodal images in medical imaging [5], [6] and tracking objects under rapidly changing lighting conditions [7].

Histograms are traditionally constructed by using each intensity sample to populate a particular histogram bin, herein referred to as standard sampling. The histogram accuracy, and hence registration accuracy, is limited by the quantisation of intensity and by the number of intensity samples available to populate the histogram. These are determined by the acquisition hardware and the number of bins in the histogram.

The authors are at Siemens Molecular Imaging Advanced Applications, 23-38 Hyth Bridge Street, Oxford, OX1 2EP, UK, and the Centre for Vision Speech and Signal Processing, University of Surrey, Guildford, GU2 7XH, UK, e-mail: \{nicholas.dowson;timor.kadir\}@siemens.com;r.bowden@surrey.ac.uk
Moddemeijer described the effects of such limitations in [8]. The number of samples further limits the number of bins, since too few samples relative to bins results in an underpopulated histogram. Without significant smoothing, usable MI values cannot be obtained from such a histogram. In addition, as the two images shift relative to one another, the discrete shifts between histogram bins result in discontinuities in the MI function, making optimisation difficult.

Underpopulated histograms may be avoided either by reducing the number of bins or by smoothing the histogram with a Parzen window after construction [9], herein referred to as Post-Parzen windowing. Thevenaz and Unser proposed Parzen Windowing during histogram construction [10] allowing fractional intensities to be used, herein referred to as InParzen Windowing. This has the useful side-effect of yielding continuous shifts in probability between bins, and hence MI, as the two images shift relative to each-other. Maes et al. proposed Partial Volume Interpolation to improve MI stability [11]. Chen and Varshney generalised this to Partial Volume Estimation (PVE) [12], which considers local neighbourhoods of more than four pixels. Rather than interpolating intensities, PVE treats intensity bins as labels. Labels corresponding to pixel values surrounding each sample point are given a weighting based on their proximity to the sample point, using the equations for bilinear interpolation in the first order case [11], [13].

These methods require the number of bins in the histogram and a kernel size to be appropriately chosen to avoid the effects of underpopulated histograms. To varying degrees all the methods, except PVE at orders greater than one, suffer from spurious local maxima [14]. Additionally, due to the use of arbitrary smoothing kernels the function maximum does not always coincide with the true alignment, i.e. there is bias. Most of the methods consider each intensity sample independently of its neighbours and assume that individual samples represent the signal behaviour in subpixel positions. PVE explicitly considers pixel neighbourhoods but only in one image; the structure in the other image is ignored. In summary, such techniques offer well engineered solutions to the joint histogram estimation problem but each has limitations either practically or theoretically.

Non-Parametric (NP) Windows, a technique proposed by Kadir and Brady [15], [16], is a signal density and distribution estimator that is founded upon the Shannon-Whittaker-Nyquist theory of sampled signals. It estimates signal statistics by directly calculating the distribution of each piecewise section 
of a signal for a given interpolation model. In contrast to previous methods, interpolation or smoothing is performed in the signal domain rather than the probability domain. Hence, the method rests upon the assumption that the signal is bandlimited and at least critically sampled - a basic assumption of standard signal sampling theory.

Aside from theoretical attractions, NP Windows also accounts for the subpixel behaviour of signals under a given interpolation model. Further, arbitrary selection of a kernel is not required and the quality of the distribution estimate is unaffected by the number of bins or the number of available samples, i.e. histogram underpopulation never occurs. NP Windows has been shown to yield accurate histograms that vary smoothly with sample point position, due to the treatment of images as continuous functions.

The benefits of NP windows make the technique attractive for application to the estimation of Mutual Information for image registration. To date, NP Windows has only been applied to marginal distributions of 2D signals and joint distributions of 1D signals. The primary contribution of this work is to extend NP Windows to obtain joint distributions of $2 \mathrm{D}$ signals. For registration, the absence of any smoothing in the probability domain should result in reduced bias (accuracy) and histogram stability, thereby improving convergence (repeatability). For tracking, the issue is mainly the accumulation of subpixel errors. Particularly in applications where no $a$ priori appearance model is available, templates are extracted on-the-fly [17], [18], and depend on the accuracy of previous registrations. Errors compound as the number of templates extracted increases, significantly affecting performance on long sequences.

A second contribution is to provide simplifications to the original NP Windows derivations, using Green's Theorem. These lead to substantial reductions in the complexity of the implementation for the case of marginal histogram estimation of $2 \mathrm{D}$ images. In particular we note that in the partial pixel extension of NP Windows, presented in [15], the large number (tens) of geometric special cases was too cumbersome to implement. Instead, Kadir and Brady resorted to a numerical implementation of their technique.

We should point out that the assumption of signal continuity in image registration applications is not always valid. For example, where the pixel intensities are regions or class labels in segmented images, NP Windows may not be applicable. For such applications successful results have been demonstrated for segmented brain scans using the approach of D'Agostino et al. [13].

Independently of this work, but subsequent to Kadir and Brady's paper [15], Rajwade et al. have also developed a similar density estimation method [19] for joint distributions. However, Rajwade's method is never used to actually register images. Moreover degeneracies in their method are solved by imposing constraints on the extent of the probability distribution, which is an approximation, whereas we explicitly solve each degeneracy.

The remainder of the paper is organised as follows. A background to NP Windows is presented in Section II. Simplifications to the NP Windows theory for 2D marginal distributions using Green's Theorem are described in Section III Section IV proposes several approaches for obtaining joint distributions of 2D signals, and discusses their various trade-offs. A number of experiments and their associated results are presented in Section V] followed by our conclusions and a discussion of future work in Section VI.

\section{BACKGROUND}

\section{A. Registration using Mutual Information}

A number of approaches to registration exist. In this work we consider the widely used approach of maximisation of Mutual Information, $I$ :

$$
\mathbf{v}_{\text {optimum }}=\arg _{\mathbf{v}} \max I\left[f_{1}(\mathbf{w}(\mathbf{x}, \mathbf{v})), f_{2}(\mathbf{x})\right]
$$

where the optimal transformation parametrised by $\mathbf{v}$ between two images, $f_{1}$ and $f_{2}$, is found. The images are functions of a spatial parameter, $\mathbf{x} \in \mathbb{R}^{2}$, and the transformation or warp function between the coordinate systems is denoted by $\mathbf{w}$. Several algorithms exist to optimise (1), e.g. Powell's method, and the Levenberg-Marquardt algorithm. A discussion of these is beyond the scope of this paper, suffice it to say that a derivative of $I$ is not provided in this work, restricting the choice of optimisation method to one that does not require a Jacobian or Hessian.

The origins of MI are in information theory [20]. It was first proposed by Shannon [21] as method to measure the amount of the shared information between two signals, with quantised amplitudes over a period of time. It is a simple extension to consider 2D images rather than 1D signals, which consist of quantised intensities over a 2D space. MI is calculated using the joint probability distribution function (PDF) of the two signals, $f_{1}$ and $f_{2}$, under consideration, with respective intensities $i_{1}$ and $i_{2}$ :

$$
I(\mathbf{v})=\sum_{i 1, i 2} p_{f_{1} f_{2}}\left(i_{1}, i_{2}, \mathbf{v}\right) \log \left(\frac{p_{f_{1} f_{2}}\left(i_{1}, i_{2}, \mathbf{v}\right)}{p_{f_{1}}\left(i_{1}, \mathbf{v}\right) p_{f_{2}}\left(i_{2}, \mathbf{v}\right)}\right)
$$

The joint PDF of $f_{1}$ and $f_{2}$ is denoted by $p_{f_{1} f_{2}}$. The marginal PDFs, $p_{f_{1}}$ and $p_{f_{2}}$, are easily obtained from the joint PDF, since $p_{f_{1}}=\sum_{i 2} p_{f_{1} f_{2}}$, and $p_{f_{2}}=\sum_{i 1} p_{f_{1} f_{2}}$. Note the treatment of $i_{1}$ and $i_{2}$ as discrete variables (or indices), indicating the finite bin-size of the histogram from which $p$ is derived. Bin-size, $\Delta f$, is the number of image intensity units represented by each bin in the histogram. Bin-size is inversely proportional to the number of bins in the histogram. For the case where each joint sample from the signals is used to populate one histogram bin once, i.e. standard sampling, the residual error was found by Moddemeijer to be approximately proportional to the square of the bin-size [8] and inversely proportional to the number of samples. One could decrease the bin-size (increase histogram size) to reduce the residual error, but at some stage the histogram becomes underpopulated, e.g. Fig. 1 , and usable MI estimates cannot be obtained. Clearly, the choice of the bin-size is dependent on the number of samples and the method for populating the histogram. This choice is not trivial, so the following subsection is dedicated to a discussion of histogram estimation methods. 


\section{B. Histogramming methods}

The simple case of a single 1D signal is considered first. One such example is plotted in Fig. 1 1 a, with amplitude $f$ as a function of position $x$. A set of sample positions with interval $\Delta x=1$ is also shown. We use the example of an invertible function as the underlying signal:

$$
f(x)= \begin{cases}100 \log (x+1) & x \in[0,10] \\ 0 & \text { elsewhere }\end{cases}
$$

In practice, the signal is then quantised by the measuring process. Quantisation occurs in both position and amplitude, e.g. for a digital camera the spatial resolution is constrained by the size of the individual Charged Coupled Device (CCD) elements, and the amplitude by the number of voltage levels used to represent the intensity of light falling upon each element. The aim of the histogramming process is to obtain the statistical properties of the underlying signal despite the inaccuracy of the quantised estimate.

For the sake of discussion, in Fig. 19, an $x$-quantisation of 1 is used, and the signal is measured exactly at each sample point. Generally, signals are not measured at single points, but integrated and normalised over each $x$-interval. This is equivalent to filtering the signal prior to point sampling; for clarity we assume that this step has already be performed. Let us further assume that an $f$-quantisation of 1 is available.

Since the underlying signal is known, a cumulative distribution function, $P$, may be directly obtained from (3) by inverting the equation, integrating and normalising to obtain:

$$
P_{f}(f)=\frac{100 e^{f / 100}-f-100}{1100-100 \log (11)-100}
$$

A histogram of given bin-size, $\Delta f$, is obtained by finding the change in $P$ between each bin-boundary $p_{f}(f)=$ $\Delta f\left(P_{f}(f+\Delta f)-P_{f}(f)\right)$. The histogram for a bin-size of 1 is shown in Fig. 11p. Of course, the underlying signal is seldom available in practice, only the set of eleven samples, which with a $\Delta f=8$, results in the underpopulated histogram in Fig. 11. Note that, although the human reader will perceive the increasing density of samples, the MI function will not be affected by this as it treats the histogram as an independent series of probability values.

Applying further $f$-quantisation, by increasing the bin-size can improve matters but only at the cost of the coarser histogram estimate in Fig. 11. The histogram may also be convolved with a Parzen window to convert local probability density into local probability, resulting in Fig. 11. This may also be thought of as modelling the uncertainty in the value of each intensity sample.

Alternatively, if a particular interpolation model is assumed, then additional samples may be obtained at positions between the original eleven basis-samples, using Monte-Carlo. The term basis-samples is used to indicate signal values that are used to obtain the interpolated values. As the number of interpolated samples is increased, the resulting PDF, in Fig. 1f, begins to better resemble the true PDF, in Fig. 1k. Notably, Nearest Neighbour interpolation would give a result like that in Fig. 1k, since no variation in intensity in intermediate positions is allowed.
Ignoring signal noise, the accuracy of the PDF is limited by two factors, the resolution of the supplied signal and the accuracy of the interpolation model. Without hardware changes, the resolution of the signal, and hence the number of basis-samples, is generally fixed. An improved interpolation model may be used if there is a known point spread function associated with the signal. In this work we assume that this is unavailable.

By increasing the sampling resolution to infinity, the most accurate possible PDF may be obtained within the above limitations. Of course, taking infinite, or a simply a sufficiently large number to achieve a prespecified accuracy using MonteCarlo techniques, is impractical. However, the equivalent problem may be solved by finding the function of $f$ over each interpolated interval between basis-samples. This technique is referred to as NP Windows. NP Windows was used to obtain the histogram shown in Fig. 1,

\section{NP Windows on single 1D signal: $f(x)$}

NP Windows may be considered to be the closed-form equivalent to obtaining infinite samples of an image using a Monte-Carlo approach for a given interpolation method. If linear interpolation is used, the equation for $f$ in the interval between two basis-samples, $\alpha$ and $\beta$, takes the form:

$$
f=a x+b \quad \mid \quad 0 \leq x<1
$$

The terms $a$ and $b$ allow a simplified equation to be used, and relate to the basis-samples as follows: $a=\beta-\alpha$, and $b=\alpha$.

The distribution of $x$, the domain of the function, is considered to be uniform between each pair of basis-samples; no particular value of $x$ is more or less likely than another:

$$
p_{x}(x)= \begin{cases}1 & 0 \leq x<1 \\ 0 & \text { elsewhere }\end{cases}
$$

Using standard probability theory, the probability of getting a particular $f$ may be obtained by using the transformation formula. This may take one of two forms [22], depending on the absolute (Jacobian) gradient of $x$ with respect to $f$, or vice-versa:

$$
p_{f}(f)=\left|\frac{\partial x}{\partial f}\right| p_{x}(x(f))=\left|\frac{\partial f}{\partial x}\right|^{-1} p_{x}(x(f))
$$

This highlights an important requirement for the methods used in this paper: the interpolation function used must be invertible. Even if the inverse (second) form of the transformation function is used, the inverse function $x(f)$ is required for substitution into the final form of (7).

Non-invertible equations can be dealt with by partitioning the function into invertible sections [16] or alternatively locally linearised and then inverted. Consideration of non-invertible interpolation models is reserved for future work.

Inverting the linear interpolation function is trivial: $x(f)=$ $\frac{f-b}{a}$, so $\frac{\partial x}{\partial f}=\frac{1}{a}$. Hence, the probability for $f$ may be calculated for each adjacent pair of basis-samples:

$$
p_{f}(f)= \begin{cases}\frac{1}{|a|} & f \in[b ; a+b)=[\alpha, \beta) \\ 0 & \text { elsewhere }\end{cases}
$$



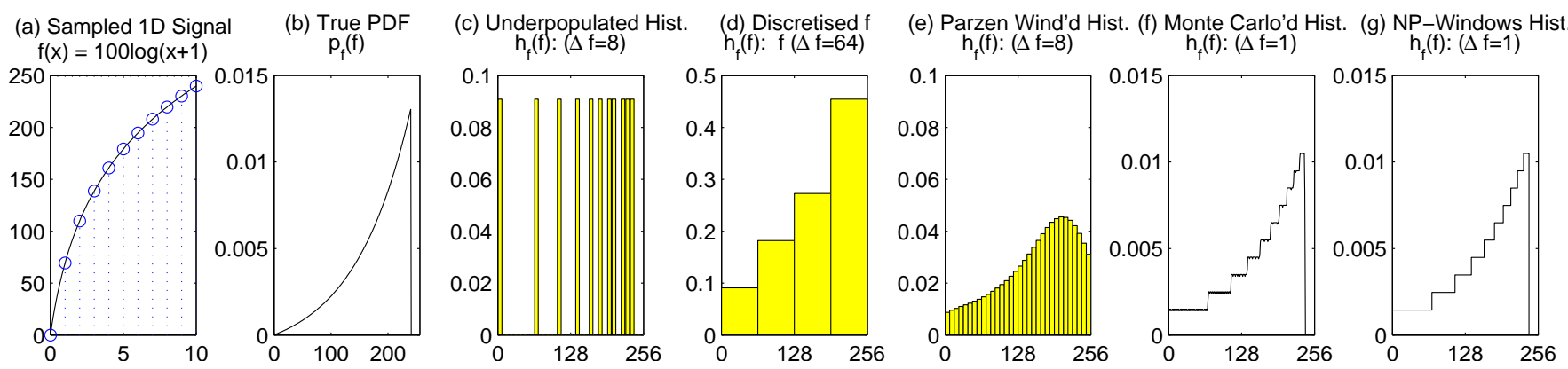

Fig. 1. Methods for extracting histograms from the 1D signal shown in $(a)$, from which eleven basis-samples (circles) are extracted. (b) The true PDF of $f(x)$ with $\Delta f$ of 1 for reference. (c) The underpopulated histogram resulting from naive histogramming. (d) Using a larger $\Delta f$ rectifies this but gives a crude approximation to (b). (e) Convolving (c) with a Parzen window improves the approximation. ( $f$ ) Using Monte-Carlo simulation to sample the signal and using linear interpolation between basis-samples gives a still closer approximation to (b). ( $g$ ) NP Windows gives the equivalent of a Monte-Carlo simulation with infinite samples. This provides the closest possible approximation to (b) obtainable with the available data.

The bounds on the non-zero portion of $f$ in $(8)$ arise from the bounds on the non-zero portion of $x$ in (6). The extent of the bounds in $f$ is $a$, so the integral of the $p_{f}$ over the domain of $f$ is 1 , an intuitively expected result.

Of course signals will comprise multiple pairs of adjacent basis-samples, hereafter referred to as neighbourhoods, each of which contributes to the overall PDF. In the example of Fig. 1, 10 such neighbourhoods exist. If $n$ is used to index each neighbourhood, $\alpha_{n+1}=\beta_{n}$, where $n \in\left[1 ; N_{n}\right] \in \mathbb{Z}$ and $N_{n}$ is the number of neighbourhoods. In standard histogramming approaches, each sample is appropriately weighted and cumulatively summed. Similarly, for NP Windows, each neighbourhood is weighted and the cumulative sum of contributions forms a histogram, as shown in Fig. 2 a. To treat each neighbourhood equally, a uniform weighting is used:

$$
\rho_{f}(f)=\frac{1}{N_{n}} \sum_{n} p_{f, n}(f)
$$

where $p_{f, n}$ indicates the PDF for neighbourhood $n$. As shown, the resulting PDF in Fig. 19 is an improvement over those obtained using standard (non-Monte Carlo) histogramming approaches and comes at an equivalent computational complexity of $O\left(N_{n}\right)$.

The PDF $\rho_{f}(f)$ may be represented in several ways, of which two are discussed here. In one representation, the PDFs for each neighbourhood are kept in a sorted list of $f$-values, each with an associated $\rho_{f}$ value. Each entry in the list indicates a position where $\rho_{f}$ changes and is valid over the range $f_{j}, f_{j+1}$, where $j$ indexes the current position in the list. Such a representation, e.g. Fig. 2p, is accurate and convenient for marginal PDFs since a binomial search suffices to find $\rho_{f}$ at a particular point in $f$. A cumulative distribution function could be similarly represented: a pair of parameters describing the linear behaviour of $P_{f}$ over each $f_{j}, f_{j+1}$ interval, is associated with each $f$-value.

Alternatively, a discretised PDF (histogram) may be used, where the $f$-values are at pre-defined, regular positions. The $\rho_{f}$ associated with each $f$-value becomes the mean probability within the $f_{j}, f_{j+1}$ interval, e.g. Fig. 2k. This is an approximation of the first representation, but offers faster lookup. The histogram in Fig. 2k may be obtained directly from Fig. 2 p

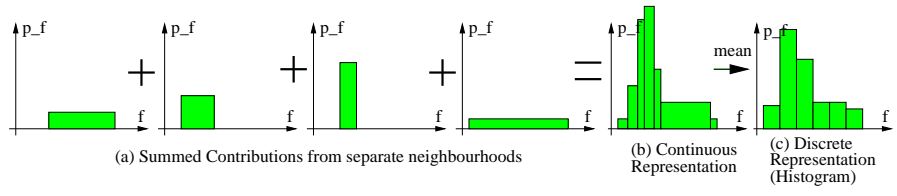

Fig. 2. Diagram of how the probability contributions from each pair of adjacent basis-samples (neighbourhood), shown in (a), are combined to form an exact representation of the PDF in (b) in the case of a single 1D signal. This simply requires a list of $f$ intervals and some parameters describing the behaviour of the PDF within each interval. A quantised representation or histogram, shown in (c), uses fixed regularly spaced intervals and the mean probability within each interval is stored, which is an approximation. The histogram is obtained by integrating (b) between each pair of bin boundaries.

by integrating between each pair of bin edges and normalising by the interval between the bins.

\section{NP Windows to estimate the joint histogram for a pair of $1 D$ signals: $f \rightarrow \mathbf{f}$}

For the joint distribution of two linearly interpolated 1D signals, two equations describe the signals: $f_{1}=a_{1} x+b_{1}$ and $f_{2}=a_{2} x+b_{2}$. These can be grouped together to form a vector, $\mathbf{f}=\left(f_{1}, f_{2}\right)$, and a more general form of the inverse transformation formula is used to determine the joint distribution where the scalar $f$ becomes a vector $\mathbf{f}$. Here the magnitude of the derivative generalises to the $L_{2}$-norm of the vector derivative as indicated by $\|\cdot\|$ :

$$
p_{f}(\mathbf{f})=\left\|\frac{\partial \mathbf{f}}{\partial x}\right\|^{-1} p_{x}(x(\mathbf{f}))
$$

In this case the partial derivative is: $\frac{\partial \mathbf{f}}{\partial x}=\left(a_{1}, a_{2}\right)$. The $L_{2}$ norm of $\frac{\partial \mathbf{f}}{\partial \mathbf{x}}$ is simply its length so the PDF becomes:

$$
p_{\mathbf{f}}(\mathbf{f})=\left\{\begin{array}{cc} 
& b_{1} \leq f_{1}<a_{1}+b_{1} \\
\frac{1}{\sqrt{a_{1}^{2}+a_{2}^{2}}} & f_{2}=\frac{a_{2}}{a_{1}}\left(f_{1}-b_{1}\right)+b_{2} \\
0 & \text { elsewhere }
\end{array}\right.
$$

As this is a joint case, the PDF has two dimensions but is constrained to a single line segment, because the two signals share the same variable upon each other. The line segment is finite because $0 \leq x<1$, resulting in the bounds on $f_{1}$ given in 11 . 
The discussion thus far has assumed that the basis-samples for $f_{1}$ and $f_{2}$ align exactly. This is not necessarily the case, and to obtain co-incident values for $f_{1}$ and $f_{2}$ at least one of the signals will need to be interpolated. We use the convention of always interpolating $f_{2}$. Hence two interpolation operations are explicitly applied: one to obtain the basis-samples in $f_{2}$ coincident with the basis-samples in $f_{1}$, and one as the interpolation model used by NP Windows. The term basis-sample refers to both $f_{1}$ and the interpolated $f_{2}$ at the corresponding position.

The additional interpolation step will be an additional source of error, particularly when the scales of the two signals are disparate. In addition, the use of interpolation to obtain the second of each basis-sample pair assumes a linear transformation between the coordinate systems of the two signals (images). This implies that only affine transformations, or some subset thereof, may be used. However in practice, the transformation need only be approximately locally linear. More precisely, considering the first order Taylor expansion of the relationship between the coordinate systems: $x_{2}=w\left(x_{1}, \mathbf{v}\right)=w(0, \mathbf{v})+$ $\frac{\partial w}{\partial x_{1}} \Delta x_{1}+O\left(\Delta x_{1}^{2}\right)$, linear interpolation may be used so long as $x_{2} \gg O\left(\Delta x_{1}^{2}\right)$. For non-linear transforms, the error due to this assumption may be kept low by strategically increasing the number of basis-samples in certain regions, e.g. Section IV-C

At this point all the elements are in place to present the NP Windows method for histogram construction. For input, two 1D signals specified as a set of values or amplitudes, along with a (locally) linear transformation. First, the intensities in $f_{2}$ corresponding to the positions of the basis-samples in $f_{1}$, are obtained using interpolation. Next, for each neighbourhood (pair of basis-samples), the parameters $a_{i}$ and $b_{i}$ for $i \in[1 ; 2] \cap$ $\mathbb{Z}$, are calculated. These parameters specify a line of constant probability in 2D PDF space. Finally, for each histogram bin that the line intersects, the probability density along the length of line contained by the bin is integrated (summed). Since the probability density is constant along the line, this is simply the proportion of the line's length within the histogram-bin which is added to the histogram bin.

All the NP Windows methods presented here follow this approach. All that varies is the probability field, the shape of the region defined (in this case lines) and hence the region of integration. This process is shown pictorially in Fig. 3

E. NP Windows to estimate the marginal for a $2 D$ image: $x \rightarrow \mathbf{x}$

Using bilinear interpolation for a single $2 \mathrm{D}$ signal, gives $f$ the form:

$$
f_{1}=a x_{1} x_{2}+b x_{1}+c x_{2}+d
$$

Each neighbourhood consists of four adjacent basis-samples from the image grid, with the following relationship to the coefficients in (12).

$$
\left(\begin{array}{l}
a \\
b \\
c \\
d
\end{array}\right)=\left(\begin{array}{cccc}
1 & -1 & -1 & 1 \\
-1 & 1 & 0 & 0 \\
-1 & 0 & 1 & 0 \\
1 & 0 & 0 & 0
\end{array}\right)\left(\begin{array}{l}
\alpha \\
\beta \\
\gamma \\
\delta
\end{array}\right)
$$

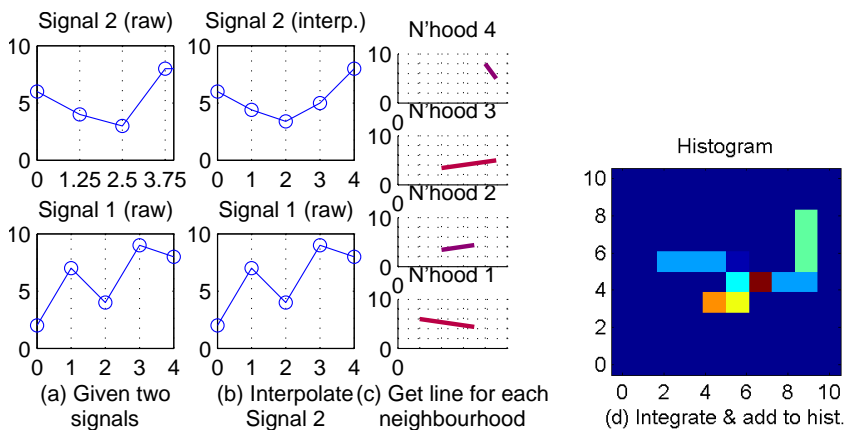

Fig. 3. Illustration of how a joint histogram is constructed using NP Windows for two 1D signals shown in (a). In (b) signal 2 is interpolated to find the values at locations corresponding to the basis-samples signal 1. In (c) each of the four neighbourhoods contributes a line of constant probability in PDFspace, which is inversely proportional to its length. In (d) these contributions are weighted and summed to form the histogram shown.

A more general form of the transformation formula is required in this case:

$$
p_{f}(\mathbf{f})=\left|\operatorname{det}\left[\frac{\partial \mathbf{x}}{\partial \mathbf{f}}\right]\right| p_{x}(\mathbf{x}(\mathbf{f}))=\left|\operatorname{det} J_{\mathbf{x} \circ \mathbf{f}}\right| p_{\mathbf{x}}(\mathbf{x})
$$

where $\left|\operatorname{det} J_{\text {xof }}\right|$ is used as a shorthand to indicate the absolute determinant of the Jacobian of $x$ with respect to $f$. The Jacobian is required to be square in order to obtain the determinant. When the dimension of $\mathbf{x}$ does not equal the dimension of $\mathbf{f}$, dummy variables can be introduced to pad the shorter vector. For this purpose $f_{2}$ is introduced, where $f_{2}=x_{1}$. The inverse equations of $x_{1}$ and $x_{2}$, in terms of $f_{1}$ and $f_{2}$, are:

$$
x_{1}\left(f_{1}, f_{2}\right)=f_{2} \quad x_{2}\left(f_{1}, f_{2}\right)=\frac{f_{1}-b f_{2}-d}{a f_{2}+c}
$$

yielding the absolute Jacobian determinant:

$$
\begin{aligned}
& \left|\operatorname{det} J_{\mathbf{x o f}}\right|=\left|\begin{array}{cc}
\frac{\partial x_{1}}{\partial f_{1}} & \frac{\partial x_{1}}{\partial f_{2}} \\
\frac{\partial x_{2}}{\partial f_{1}} & \frac{\partial x_{2}}{\partial f_{2}}
\end{array}\right| \\
& =\left|\begin{array}{cc}
0 & 1 \\
\frac{1}{a f_{2}+c} & \frac{-b\left(a f_{2}+c\right)+a\left(b f_{2}-f_{1}+d\right)}{\left(a f_{2}+c\right)^{2}}
\end{array}\right|=\frac{1}{\left|a f_{2}+c\right|}
\end{aligned}
$$

The result is the 2D PDF in (eq:transformationGeneral) becomes:

$p_{f 1, f 2}=\left\{\begin{array}{cc} & 0 \leq f_{2}<1 \\ \frac{1}{\left|a f_{2}+c\right|} & b f_{2}+d \leq f_{1}<(a+b) f_{2}+c+d \\ 0 & \text { elsewhere }\end{array}\right.$

where the bounds on $f_{1}$ and $f_{2}$ arise from those on $\mathbf{x}$, as they did in the joint $1 \mathrm{D}$ case. Only one signal exists, so the marginal PDF $p_{f 1}$ must be obtained by integrating out $f_{2}$. As shown in Fig. 4, this implies computing the integral over the shaded area bounded by four lines. The result is three equations for the three ranges where different pairs of bounds apply. The use of a discrete PDF (histogram) with prespecified bin-edges introduces further integration limits, so the probability within each bin may be calculated. The geometric arrangement of the four boundaries in (17) depends upon the bilinear coefficients of (12). There are 24 geometric arrangements for the bounding lines. In the original NP Windows approach each of the 24 


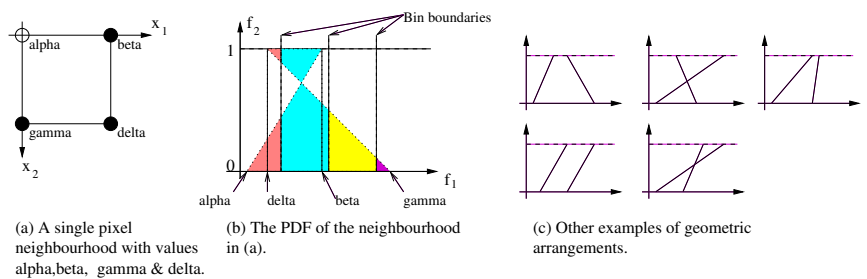

Fig. 4. Integrating out $f_{2}$ for single bilinearly interpolated image. (a) shows a single pixel neighbourhood with intensities at the basis-samples points of $(\alpha, \beta, \gamma, \delta)$. These form the area of integration (shaded) contained between the boundary lines shown in (b). The differently shaded regions indicate how the region is split to populate separate histogram bins. This is just one of 24 possible arrangements depending on the neighbourhood intensities. (c) shows five further examples of arrangements of the bounding lines.

cases was dealt with separately [15], [16], which was timeconsuming and error-prone to implement. The next section shows how Green's Theorem can be used to substantially reduce the cases that need to be considered.

\section{APPLYING GREEN'S THEOREM TO BILINEAR INTERPOLATION FOR A SINGLE IMAGE}

Green's Theorem allows an area integral like that shown in Fig. 4 to be converted into the line integral defining the boundary of the area being integrated:

$$
\iint_{\text {region }}\left[\frac{\partial g_{2}}{\partial f_{1}}-\frac{\partial g_{1}}{\partial f_{2}}\right] \mathrm{dArea}=\oint_{\text {curve }} g_{1} \mathrm{~d} f_{1}+g_{2} \mathrm{~d} f_{2}
$$

The curve of integration must be closed and is by convention anti-clockwise. Although (18) allows two functions to be chosen, 177 has only one term so we let $\frac{\partial g_{2}}{\partial f_{1}}=0$ and $\frac{\partial g_{1}}{\partial f_{2}}=-\left|\operatorname{det} J_{\mathbf{x o f}}\right|$. Integrating the expression for the single $2 \mathrm{D}$ case in 16 to obtain $g_{1}$ yields:

$$
g_{1}=-\frac{1}{a} \log \left(\left|a f_{2}+c\right|\right)+\text { const }
$$

The curve in this case consists of four lines. Hence the final form of 18 is a sum of four line integrals: $\oint_{\text {curve }} g_{1} \mathrm{~d} f_{1}=$ $\sum_{j} \int_{\text {line } j} g_{1} \mathrm{~d} f_{1}$. The constant term in 19 cancels out since the integral is definite. Each line may easily be parametrised as follows: $f_{2}=m_{j} f_{1}+n_{j}$, where $j$ indexes the current edge. This also allows $f_{2}$ to be substituted out of the equation, giving the probability contained within a region or part thereof:

$$
\begin{aligned}
& \int_{\text {line } j} \frac{1}{a} \log \left(a\left(m_{j} f_{1}+n_{j}\right)+c\right) \mathrm{d} f_{1}=\frac{1}{m_{j} a^{2}} . \\
& \cdot\left[\left(a\left(m_{j} f_{1}+n_{j}\right)+c\right) \log \left(\left|a\left(m_{j} f_{1}+n_{j}\right)+c\right|\right)-m_{j} a f_{1}\right]
\end{aligned}
$$

However several geometric special cases need to be considered, since without care 20 can become indeterminate. Each degeneracy is tabulated in Figure 5 with a description of its geometry in PDF space, the behaviour the intensity and its mathematical form. The derivations, shown in the fourth column, will replace 20 for these special cases. Examples of the degeneracies are also given in Fig. 6, to give a visual interpretation. The derivations were obtained by substituting the degeneracy in algebraic form (e.g. $a=0)$ into (17) or (19) and following the steps shown above. In some cases, substitution into 20 and the use of L'Hopital's rule was
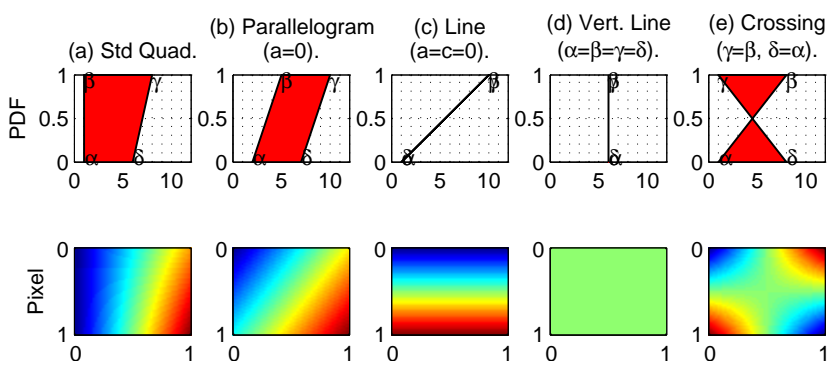

Fig. 6. Examples of the singularities described in Figure 5 The top row shows the form of the shape of the region in PDF space, and the bottom row shows the image intensities within the neighbourhood forming the above PDF region.
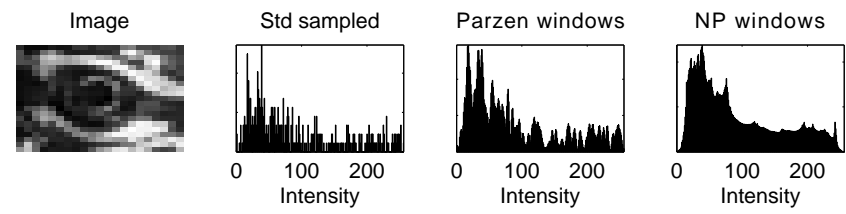

Fig. 7. Some examples of histograms obtained from the image on the far left using different sampling methods. From left to right these are: standard sampling, Parzen windowing and NP Windows. Notice the discrete changes between bins in standard sampling, where one expects a PDF to be smooth. Parzen windowing gives an improved estimate by explicitly accounting for the uncertainty in intensity, but this is simply a convolved version of the standard sampled histogram, so it also exhibits sudden changes and under-sampling in certain regions. NP Windows, on the other hand, exhibits a smooth nature where the statistics in all regions are well described.

necessary. The cases for horizontal and vertical lines of integration occur frequently, due to the bounds on $f_{2}$ and the use of bin-boundaries (shown by the differently shaded regions in Fig. 47.

The proposed method yields the same results as [15], but with substantially less complexity. Only the special cases listed in Figure 5 required separate implementation, rather than the 24 cases in the previous implementation [15]. Several histograms obtained using different sampling methods, shown in Fig. 7. demonstrate the accuracy of the proposed approach.

\section{The JoInt 2D CASE}

\section{A. Bilinear Interpolation}

Bilinear interpolation would perhaps be the intuitive generalisation to $2 \mathrm{D}$ from a $1 \mathrm{D}$ linearly interpolated signal. The equations for the joint bilinearly interpolated case are the same as before, with the addition of an index for each image, $l$ :

$$
f_{l}=a_{l} x_{1} x_{2}+b_{l} x_{1}+c_{l} x_{2}+d_{l}
$$

In contrast to the 2D marginal case, here the number of $x$-dimensions equals the number of $f$-dimensions. Hence, the Jacobian is naturally square and a determinant may be obtained directly. However, due to the cross term in the above equation, two possible pairs of inverse functions are yielded. Both pairs take the form: $x_{i}\left(f_{1}, f_{2}\right)=\phi\left(f_{1}, f_{2}, 1\right)+\sqrt{\phi\left(f_{1}, f_{2}, 2\right)}$, where $\phi(\ldots, \epsilon)$ indicates a polynomial of degree $\epsilon$, the absence of which indicates degree 1 . This concise notation is used for clarity. Both pairs of solutions give the same result for 


\begin{tabular}{|c|c|c|c|c|}
\hline Geometric Description & Intensity Description & Mathematically & \multicolumn{2}{|c|}{ Form of $\int_{\text {curve } j} g_{1} \mathrm{~d} f_{1}$} \\
\hline $\begin{array}{l}\text { Line of integration } \\
\text { is vertical. }\end{array}$ & See Fig. 6 . & $m_{j}=\infty$ & \multicolumn{2}{|l|}{0} \\
\hline $\begin{array}{l}\text { Line of integration } \\
\text { is horizontal. }\end{array}$ & & $m_{j}=0$ & $p_{f 1}=$ & $\begin{array}{rr}\frac{1}{a} \log (a+c) f_{1} & f_{2}=1 \\
\frac{1}{a} \log (c) f_{1} & f_{2}=0\end{array}$ \\
\hline $\begin{array}{l}\text { Boundaries form a } \\
\text { parallelogram. }\end{array}$ & $\begin{array}{l}\text { Gradient is constant. } \\
\text { See Fig. } 6 \text { p. }\end{array}$ & $a=0$ & \multicolumn{2}{|c|}{$\frac{1}{2 c}\left(m_{j} f_{1}^{2}+2 n_{j} f_{1}\right)$} \\
\hline $\begin{array}{l}\text { Boundaries form a } \\
\text { line. }\end{array}$ & $\begin{array}{l}\text { Gradient is constant } \\
\text { and axis aligned. } \\
\text { See Fig. 6e. }\end{array}$ & $a=c=0$ & \multicolumn{2}{|l|}{$\frac{1}{\beta-\alpha}$} \\
\hline $\begin{array}{l}\text { Boundaries form a } \\
\text { vertical line. }\end{array}$ & $\begin{array}{l}\text { Gradient is zero. } \\
\text { See Fig. } 6 \mathrm{~d} .\end{array}$ & $a=b=c=0$ & $p_{f 1}=$ & $\begin{array}{ll}1 & f_{1}=\delta \\
0 & \text { elsewhere }\end{array}$ \\
\hline $\begin{array}{l}\text { Singularities at some points } \\
\text { in line of integration } \\
\text { e.g. hourglass centre. }\end{array}$ & See Fig. 6 e. & $\begin{array}{l}a\left(m_{j} f_{1}+n_{j}\right)+ \\
+c=0\end{array}$ & \multicolumn{2}{|l|}{0} \\
\hline
\end{tabular}

Fig. 5. Special cases when integrating Jacobian for a single bilinear interpolation image

$\left|\operatorname{det} J_{\mathbf{x} \circ \mathbf{f}}\right|:$

$$
\begin{aligned}
\left|\operatorname{det} J_{\mathbf{x} \circ \mathbf{f}}\right| & =\left[\left(b_{2} c_{1}-b_{1} c_{2}+a_{2}\left(d_{1}-f_{1}\right)-a_{1}\left(d_{2}-f_{2}\right)\right)^{2}\right. \\
- & \left.4\left(a_{2} c_{1}-a_{1} c_{2}\right)\left(b_{2}\left(d_{1}-f_{1}\right)-b_{1}\left(d_{2}-f_{2}\right)\right)\right]^{-\frac{1}{2}}
\end{aligned}
$$

As for the marginal 2D case, the valid region in the PDF is a polygon bounded by four edges. However here, the $f_{2^{-}}$ coordinates are no longer 0 and 1 , but take on the coordinates defined by the basis-samples of the second image, i.e. $\left(\alpha_{1}, \alpha_{2}\right)$, $\left(\beta_{1}, \beta_{2}\right),\left(\gamma_{1}, \gamma_{2}\right)$, and $\left(\delta_{1}, \delta_{2}\right)$.

To calculate the histogram, the total probability over a particular bin covered by the polygon may be calculated by integrating (22) directly. This is done by performing a series of piecewise integrals with integration limits set at $f_{1}$ and $f_{2}$ values corresponding to the bin boundaries. The limits occur on both $f_{1}$ and $f_{2}$ and numerous possible geometric arrangements between the histogram bins and the polygon vertices must be considered. However, as with the marginal case, Green's theorem allows us to deal with the various geometric configurations. In this case, it is used to evaluate the integral $\iint_{\text {Region }}\left|\operatorname{det} J_{\text {xof }}\right| \mathrm{d} f_{2} \mathrm{~d} f_{1}$

Since $\left|\operatorname{det} J_{\mathbf{x o f}}\right|$ is a square-root polynomial, the various terms cannot be separated into the two terms of the Green's theorem equation, 18. So either $\frac{\partial g_{2}}{\partial f_{1}}=\left|\operatorname{det} J_{\mathbf{x o f}}\right|$ or $\frac{\partial g_{1}}{\partial f_{2}}=$ - $\left|\operatorname{det} J_{\text {xof }}\right|$, with other term set to zero. This simply changes whether the integral is taken with respect to $f_{2}$ and then $f_{1}$ or vice-versa. The final result is unaffected. We use the case where $\frac{\partial g_{1}}{\partial f_{2}}=-\left|\operatorname{det} J_{\mathbf{x o f}}\right|$, so $\left|\operatorname{det} J_{\mathbf{x o f}}\right|$ must first be integrated with respect to $f_{2}$. As with the marginal, substitution of $f_{2}=m_{j} f_{1}+n_{j}$ is performed, where $j$ indexes each line segment defining the valid region. Finally, the integral along each line segment is performed. Note that two integration steps are required in a similar manner to (19) and (20).

The result of the above (double) integration is an equation containing more than 100 terms and factors, and had to be obtained using Mathematica. For interest the form of the equation is:

$$
\begin{array}{r}
\rho=k_{1} \sqrt{\phi\left(f_{1}, 2\right)}+\phi\left(f_{1}\right)\left(k_{2}+\log \left[\phi\left(f_{1}\right)+k_{3} \sqrt{\phi\left(f_{1}, 2\right)}\right]\right. \\
+k_{4} \log \left[\phi\left(f_{1}\right)+k_{5} \sqrt{\phi\left(f_{1}, 2\right)}\right]+k_{6} \log \left[\frac{\phi\left(f_{1}\right)+k_{7} \sqrt{\phi\left(f_{1}, 2\right)}}{\phi\left(f_{1}\right)}\right]
\end{array}
$$

Clearly the implementation of $(23)$, although giving a precise result, would be too slow for practical use. Several hundred operations would be required just to initialise the constants for each polygon. In addition, since a histogram approximation to the PDF is being used, the polygon contributed by each neighbourhood would need to be sliced up into the separate bins it overlaps. Several tens of operations would be required for every bin overlapped in this way.

We note that the main source of the algebraic complexity in the inverted bilinear equations is the cross-term between $x_{1}$ and $x_{2}$ in 21). Removing this term allows the development of a much simpler implementation as discussed in the next section.

\section{B. Half-bilinear Interpolation}

The removal of the cross-term in (21) means that only three coefficients are required fit the interpolating function in each section:

$$
f_{l}=a_{i} x_{1}+b_{i} x_{2}+c_{i}
$$

Only three basis-samples are required to specify the three coefficients, with the relationship:

$$
\left(\begin{array}{l}
a \\
b \\
c
\end{array}\right)=\left(\begin{array}{ccc}
-1 & 1 & 0 \\
0 & -1 & 1 \\
1 & 0 & 0
\end{array}\right)\left(\begin{array}{l}
\alpha \\
\beta \\
\gamma
\end{array}\right)
$$

To obtain the pairs of basis-sample triplets, the image lattice may be divided up into neighbourhoods of regular $45^{\circ}$ right-angled, non-overlapping triangles. Hence this form of interpolation is referred to as half-bilinear interpolation. The convention followed is to refer to the basis-sample lying at the $90^{\circ}$ apex of the triangle as $\beta$ and the remaining two basis samples as $\alpha$ and $\gamma$. The pair of inverse functions arising from (24) is:

$x_{1}=\frac{c_{1} b_{2}-b_{1} c_{2}-b_{2} f_{1}+b_{1} f_{2}}{b_{1} a_{2}-a_{1} b_{2}} \quad x_{2}=\frac{c_{1} a_{2}-a_{1} c_{2}-a_{2} f_{1}+a_{1} f_{2}}{-b_{1} a_{2}+a_{1} b_{2}}$

In this case the resulting $\left|\operatorname{det} J_{\mathbf{x o f}}\right|$ is:

$$
\begin{aligned}
\left|\operatorname{det} J_{\mathbf{x o f}}\right| & =\left|\begin{array}{ll}
\frac{\partial x_{1}}{\partial f_{1}} & \frac{\partial x_{1}}{\partial f_{2}} \\
\frac{\partial x_{2}}{\partial f_{1}} & \frac{\partial x_{2}}{\partial f_{2}}
\end{array}\right|=\left|\begin{array}{ll}
\frac{-b_{2}}{a_{1} b_{2}-b_{1} a_{2}} & \frac{b_{1}}{a_{1} b_{2}-b_{1} a_{2}} \\
\frac{a_{1} b_{2}-b_{1} a_{2}}{a_{1}} & \frac{a_{1}}{a_{1}-b_{1} a_{2}}
\end{array}\right| \\
& =\frac{1}{\left|a_{1} b_{2}-b_{1} a_{2}\right|}
\end{aligned}
$$


The absolute determinant of the Jacobian for each neighbourhood (a pair of basis-sample triplets) is constant. The area of the triangle defined by the points $\left(\alpha_{1}, \alpha_{2}\right),\left(\beta_{1}, \beta_{2}\right)$, and $\left(\gamma_{1}, \gamma_{2}\right)$, is $\frac{1}{2}\left[\left(\beta_{1}-\alpha_{1}\right)\left(\gamma_{2}-\beta_{2}\right)-\left(\gamma_{1}-\beta_{1}\right)\left(\beta_{2}-\alpha_{2}\right)\right]=$ $\frac{1}{2}\left(a_{1} b_{2}-b_{1} a_{2}\right)$. Multiplying the probability within the triangle by its area yields a total probability of $\frac{1}{2}$, which is an intuitively correct result, since only half of one pixel has been considered.

As before, in (9), the individual contributions of each neighbourhood are weighted to normalise them, and summed to obtain the overall PDF, $\rho_{\mathbf{f}}$. In this case, however, the spatial variable, $\mathbf{x}$, and intensity variable, $\mathbf{f}$, are $2 \mathrm{D}$. Note that here, neighbourhoods are single pairs of triangles and hence there are two neighbourhoods per pixel pair:

$$
\rho_{f}(\mathbf{f})=\sum_{n=1}^{N_{n}} w_{n} p_{\mathbf{f}, n}(\mathbf{f})=\sum_{n=1}^{N_{n}} w_{n}\left|\operatorname{det} J_{\mathbf{x} \circ \mathbf{f}, n}\right| p_{\mathbf{x}, n}(\mathbf{x}(\mathbf{f}))
$$

where $w_{n}$, the weighting of each neighbourhood's contribution is $\frac{2}{N_{n}}$ for triangular neighbourhoods. The correction factor of two would not be required if each pixel was counted as a neighbourhood.

Since the PDF contribution by each neighbourhood, $p_{\mathbf{f}, n}$, is constant and bounded within a triangle, the final histogram, $\rho$, can be obtained from the PDF equations using standard triangle rendering techniques from Computer Graphics [23], [24]. Such techniques offer high speed and precision. The approach is as follows: the histogram is considered an "image" whose bins are individual "pixels", and the probability in each bin is the image "intensity". Each neighbourhood corresponds to a triangle to be rendered on this image. However, unlike standard rendering, triangles are added to the histogram rather than replacing the underlying values. A brief outline of the rendering algorithm is given in Fig. 8 .

For bins at the edge of the triangle, the partial bin coverage is calculated [25]. Computing the coverage requires considering the polygon formed by the edges of a bin and detecting whether or not each polygon vertex is "below" a particular triangle edge. Vertices that are "below" every triangle edge are inside the triangle and retained. Vertices that are outside the triangle need to be sliced off. Slicing is done by calculating the intercept between the two polygon edges connected to the outside vertex and triangle edge the vertex was "above". These two intercepts are used to replace the "outside" vertex. So the polygon grows by one vertex every time this occurs. The result is a "sliced" polygon. The coverage is now simply the area of the polygon, which is calculated using the standard polygon formula [26], [27]:

$\frac{1}{2}\left[u_{1}\left(v_{2}-v_{1}\right)+u_{2}\left(v_{3}-v_{1}\right)+u_{3}\left(v_{4}-v_{2}\right)+\cdots+u_{m}\left(v_{1}-v_{m-1}\right)\right]$

where $u$ and $v$ respectively indicate $f_{1}$ and $f_{2}$ coordinates for each vertex and $m$ is the number of vertices in the sliced polygon.

The cost of evaluating the histogram is approximately dependent on the number of neighbourhoods, $N_{n}$, and the mean absolute gradient, i.e. approximately $O\left(\overline{\frac{\partial \mathbf{f}}{\partial \mathbf{x}}} N_{n}\right)$, since the larger the gradient, the larger the area of each triangle to
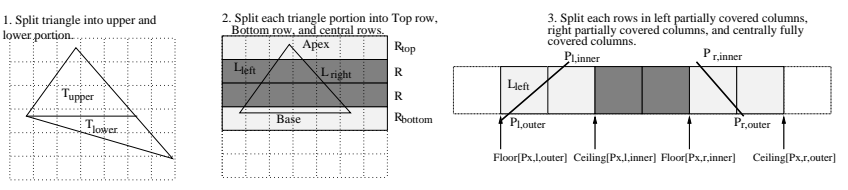

Fig. 8. Outline of triangle rendering algorithm used to render probabilities of individual neighbourhoods to a PDF. In step 1 the triangle is split into two halves using the middle $f_{2}$ value. Each triangle is rendered separately by considering each row. The fractional coverage of the row is used to weight the result from stage 3 where each column, within the row, is rendered. This involves splitting the row into the left partially covered portion, the central portion and the right partially covered portion. The partial coverage is calculated on the left and right sides and the central regions are flood filled. See [23], [24] for more details.
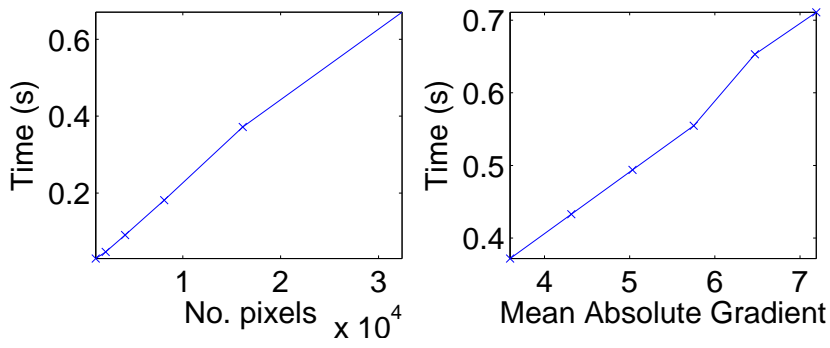

Fig. 9. Tests showing that NP Windows is $O\left(\overline{\frac{\partial \mathbf{f}}{\partial \mathbf{x}}} N_{n}\right)$. (a) Shows that NP Windows is linearly proportional to the number of neighbourhoods. (b) shows that NP Windows is linearly proportional to the mean absolute gradient.

be rendered. This was verified using two tests. In the first test two corresponding high resolution images (with and without specularities) were subsampled to vary the number of pixels from approximately 1000 to 32000 pixels. In the second test the intensities of the images were multiplied by factors ranging from 1 to 2 to vary the mean absolute gradient. The time to compute a histogram using NP Windows was averaged over ten runs in each case. The results of the tests are plotted in Fig. 9. The bulk of the computational cost is in evaluating the anti-aliasing calculations at the edges of each triangle, since simple floodfilling is used in the central part of each triangle.

Two special cases occur: when the arguments (or directions) of the intensity gradient vectors in the corresponding pixels of both images is the same, the triangle collapses into a line. Lines are easily detectable, since their area is zero. Likewise where both images have zero gradient, the triangle collapses into a point. Points may be detected by the fact that they have a perimeter of zero. Points are modelled as unit impulses, so the appropriate bin in the PDF is simply incremented with $\frac{1}{N_{n}}$. Since the intensity distribution is constant, lines are normalised by their length and split into segments wherever they intersect bin boundaries. Bins are incremented by the proportion of each line's length they contain. For points and lines $w_{n}=\frac{1}{N_{n}}$, since the integral of their contribution is 1 , unlike triangles. The three possible geometric cases are summarised in Figure 10. and some illustrative examples of each case are shown in Fig. 11 .

The NP Windows process for constructing a joint histogram from a pair of $2 \mathrm{D}$ images using half-bilinear interpolation is similar to the process described in Fig. 3 and the surrounding text in Sec. II-D. In this case $f_{2}$ is no longer constrained to be 0 or 1 , and an integral is performed over regions rather than 


\begin{tabular}{|c|c|c|c|c|}
\hline Geometric & Description & Intensity Description & Mathematically & $p_{\mathbf{f}}$ \\
\hline Triangle. S & See 11 a. & Arg of gradient different in $f_{1}$ and $f_{2}$. & $a_{1} b_{2} \neq a_{2} b_{1}$ & $\frac{1}{\left|a_{1} b_{2}-a_{2} b_{1}\right|}$ \\
\hline Line. See & $11 \mathrm{p}$. & Arg of gradient same in $f_{1}$ and $f_{2}$. & $a_{1} b_{2}=a_{2} b_{1}$ & $\begin{array}{c}{\left[\left(\min f_{1}-\max f_{1}\right)^{2}+\right.} \\
\left.+\left(\arg _{f_{2}} \min f O 1-\arg _{f_{2}} \max f_{1}\right)^{2}\right]^{-\frac{1}{2}}\end{array}$ \\
\hline Point. See & 11 . & Gradient is zero in $f_{1}$ and $f_{2}$. & $a_{1}^{2}+a_{2}^{2}+b_{1}^{2}+b_{2}^{2}=0$ & $\begin{cases}1 & \mathbf{f}=\left(\alpha_{1}, \alpha_{2}\right) \\
0 & \text { elsewhere }\end{cases}$ \\
\hline
\end{tabular}

Fig. 10. Special cases for a joint half-bilinear interpolation image. Arg stands for argument direction.
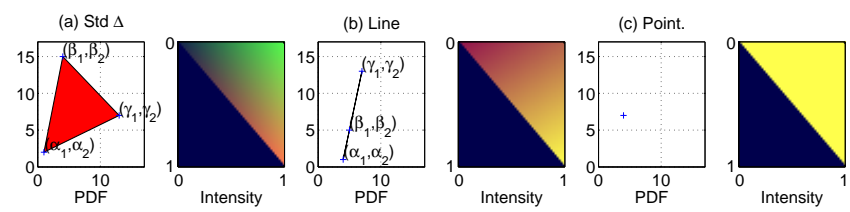

Fig. 11. Examples of the degeneracies described in Figure 10 The top row shows the form of the shape of the region in PDF space, and the bottom row shows the image intensities within the neighbourhood forming the above PDF region. The intensities of image 1 and 2 are defined by redness and greenness respectively.

lines to discretise the PDF into a histogram. The probability value within the regions of integration is always constant. Hence for triangles, lines and points, the integral respectively consists of calculating an area, length, or takes the value one. To illustrate, the process of obtaining the PDF contribution from a single pair of neighbourhoods (in a cell of four basissamples) is illustrated in Fig. 12. Each neighbourhood forms a single triangle in the PDF, and the vertex coordinates in the joint PDF directly correspond to the intensity values in the two images. The probability over each triangle sums to $\frac{1}{2}$, so the smaller triangle has a higher weighting in Fig. 12k.

Using the alternative diagonal to split the four basis-samples in Fig. 12 into two neighbourhoods, results in the alternate diagonal splitting the quadrilateral into two triangles in the joint PDF, with appropriate updates to the triangle probabilities so that they sum to one.

The fact that there are two choices of diagonal when splitting each cell defined by four intensities in the image implies some ambiguity, since there are $2^{\left(N_{x 1}-1\right)\left(N_{x 2}-1\right)}$ ways in which the image may be split up. $N_{x 1}$ and $N_{x 2}$ respectively indicate the width and height, in pixels, of the image being evaluated. This ambiguity may be removed by introducing an additional basis-sample in the centre of each pixel to split it into four neighbourhoods (rather than the usual two). However, this comes at some computational cost: a mean time $350 \mathrm{~ms}$ to evaluate the MI for two $100 \times 100$ images versus a mean time of $250 \mathrm{~ms}$ when using a single diagonal split. The three pixel splitting methods, namely: "\split", "/ split", and "X split", were compared by measuring the MI values as two images were rotated and the $x_{1}$ offset between them was varied. As shown in Fig. 13, the differences in MI value between the methods were insignificant.

\section{Resolution Aware PDF estimation}

As with other joint histogram estimation methods, NP Windows requires an additional interpolation operation to obtain coincident points in a pair of images. As mentioned

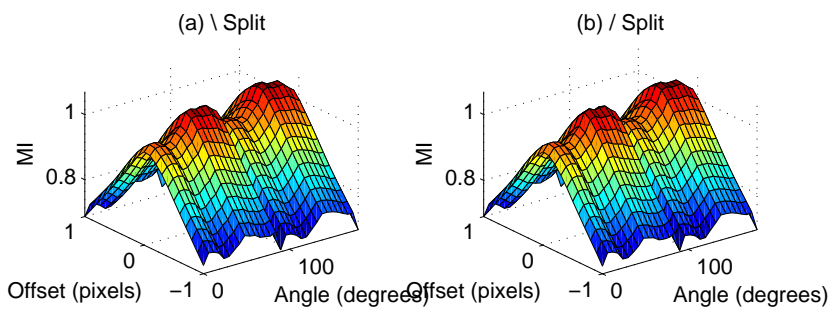

(c) X Split

(d) \Split - X Split

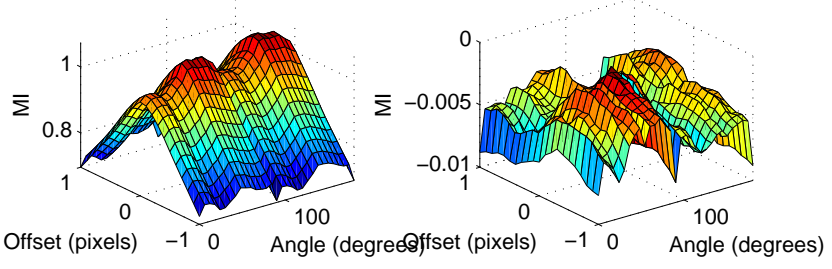

Fig. 13. Mutual Information values obtained when co-rotating two images and varying the $x_{1}$ offset between them for the three proposed methods for splitting pixels into neighbourhoods for half-bilinear interpolation, namely: (a) "\split", (b) "/ split", and (c) "X split". The images were pre-registered images of the same scene (See Fig. 21p) with strong specularities under varying lighting conditions. (d) shows the difference between (a) and (c), which was minor. Similar differences between (b) \& (c) and (a) \& (b) were obtained.

in Section II-D this means that two interpolation methods are used: a signal model for NP Windows, and to obtain the second of each basis-sample pair; these methods need not be the same. In the experiments reported here, bilinear interpolation was used to obtain coincident points, and halfbilinear interpolation was used as the NP Windows model. The use of interpolation to map points from one image to the other does not introduce significant errors as long as the template and the reference images have similar scales. However, at widely differing scales, one of the images becomes sparsely sampled and the model of it becomes inaccurate, e.g. the situation shown in Fig. 14a. Naturally, this also negatively impacts upon the PDF estimate. To overcome this problem, a resolution-aware method to obtain basis-samples was used.

The resolution-aware method uses a locally-irregular lattice to obtain each neighbourhood. The lattice points in both images are used to determine basis-sample positions while maintaining rectangular regions. This is illustrated in Fig. 14 b. Each contribution to the histogram is weighted by the neighbourhood's area relative to that of the template, $A_{n}$, so (28) becomes:

$$
\rho_{f}(\mathbf{f})=\sum_{n=1}^{N_{n}} A_{n}\left|\operatorname{det} J_{\mathbf{x} \circ \mathbf{f}, n}\right| p_{\mathbf{x}, n}\left(\mathbf{x}_{n}(\mathbf{f})\right)
$$



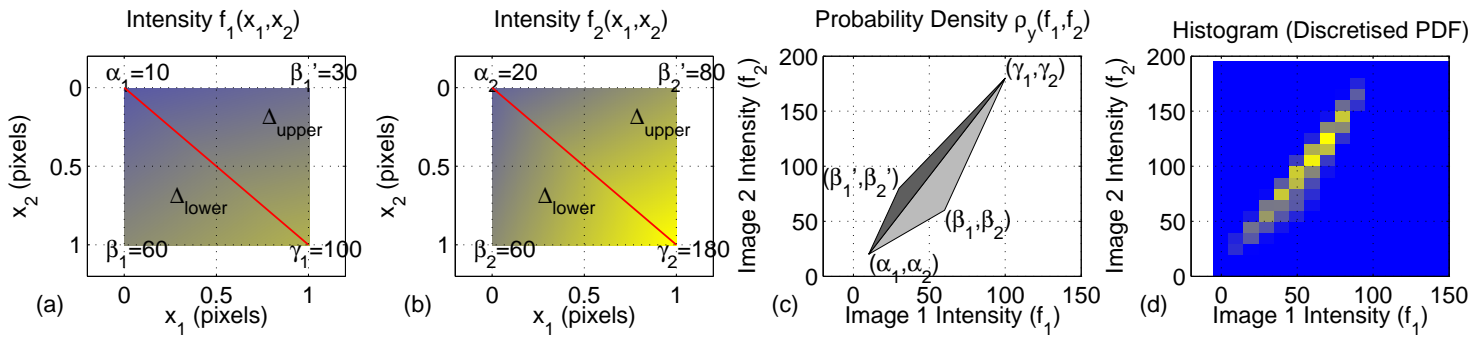

Fig. 12. (a) \& (b) Interpolated intensities for two images: respectively $\mathbf{f}_{1}(\mathbf{x})$, and $\mathbf{f}_{2}(\mathbf{x})$, where blue indicates low intensity and yellow indicates high intensity. Half bilinear interpolation is used, requiring each pixel to be split into two triangles (Split by the red line). Each corresponding triangle pair in (a) and (b) describes a triangle of uniform probability in the joint PDF of intensity, shown in (c). For each triangle in the joint PDF, the probability must integrate to one, hence the smaller triangle has a higher weight, indicated by the darker shade of gray. Note how the vertices of the two triangles in (c) correspond to intensity values in (a) and (b) respectively. Note also, that the choice of diagonal in (a) and (b), will simply change the shared diagonal between the two triangles in the joint PDF. (d) The proportional coverage of the triangle over each histogram bin is calculated and cumulatively added.
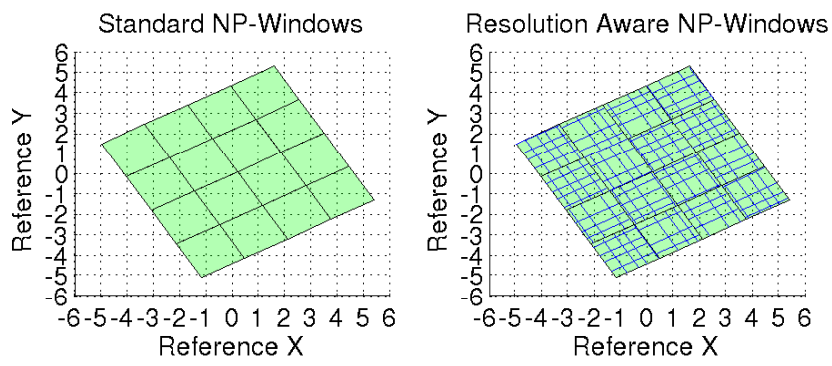

Fig. 14. Template coordinate system (green region) warped into the reference image coordinate system. As shown the lattice points of each coordinate system generally will not line up, so interpolation is required to obtain the reference intensities. (a) In standard NP Windows, a regular lattice is used for sampling. If the scales of the images are similar, the underlying reference image will be well modelled. However if their scales become too disparate, the reference image becomes too sparsely sampled to accurately model its statistics. (b) The resolution aware implementation uses a locally irregular lattice to ensure that every reference pixel within the template's bounds is sampled. Hence the reference image statistics are always correctly modelled.

The computational cost is approximately quadrupled when the relative scale of the template and the reference image is one. Otherwise, the cost is dependent on the image with the higher resolution (i.e. with the lower relative scale) in the reference coordinate system.

The algorithm to obtain lattice points in both images is an extension of the double for-loop across each dimension in $\mathbf{x}$ that is normally used. For linear transformations, each pixel-sized region (one pair of triangular neighbourhoods) in the template defines a quadrilateral in the reference image. Let $\mathbf{x}_{\mathbf{w}}=\left(x_{\mathbf{w} 1}, x_{\mathbf{w} 2}\right)=\mathbf{w}(\mathbf{x}, \mathbf{v})$, be the warped position (in the the reference image) of a location, $\mathbf{x}=\left(x_{1}, x_{2}\right)$, in the template. The minimum and maximum $x_{\mathbf{w} 1}$ and $x_{\mathbf{w} 2}$ values between the quadrilateral vertices are found. All pairs of integer $x_{\mathbf{w} 1}$ and $x_{\mathbf{w} 2}$ values between the minima and maxima are found. These integer values are then inverse transformed back into the template coordinate system. The points within the original square pixel-sized region of the template are retained, and the remainder neglected. Two sorted lists of unique $x_{1}$ and $x_{2}$ values are extracted from the retained points (including the corners of the square pixel-sized region). The pair of lists defines a local irregular lattice within the square pixel-sized region. An inner pair of for loops, which traverses the rectangles defined by the locally irregular lattice is then used to populate the histogram.

\section{EXPERIMENTS AND RESULTS}

\section{A. Bias and Convergence}

To investigate the performance of NP Windows in estimating $\mathrm{MI}$ in terms of bias and convergence, it was compared to the current state of the art MI estimation methods. In all, six methods were compared: NP Windows, resolution aware NP Windows, standard sampling, Post-Parzen windowing with $3 \times 3$ Gaussian kernel, third order In-Parzen windowing using a second order B-Spline [10], and third order partial volume estimation [12]. Since derivatives for NP Windows are not yet available, optimisation was performed using Powell's Direction Set method [28]. In this set of tests MI was maximised over two translation parameters. Bilinear interpolation was used to obtain reference image values corresponding to template lattice points, and a half-bilinear interpolation model was used for the standard and resolution aware NP Windows methods.

Testing was performed using eight sets of high resolution data (2560x1920 pixels), shown in Fig. 15. In each case the full image was used as a reference and two small sub-regions were selected as templates to be matched to the original. All the images were then blurred with a normalised $12 \times 12$ top hat function and down-sampled by a factor of 12 in each dimension. The two templates extracted from each image differed slightly. The first template was chosen to align exactly with the lattice of the down-sampled reference, and hence had intensities exactly matching those in the reference image when correctly aligned. The second template was offset from the first by $\left(+\frac{1}{3},+\frac{1}{3}\right)$ of a (down-sampled) pixel, preventing an exact match.

The methods were compared using two error measures: bias and convergence. It is well known that different sampling methods create artifacts in the function surface of MI [14]. Some artifacts can cause the maximum to shift away from its "true" position. This is referred to as bias. The use of a high resolution image and the subsequent sub-sampling process allowed the determination of the true template location despite the intensities not exactly matching. 


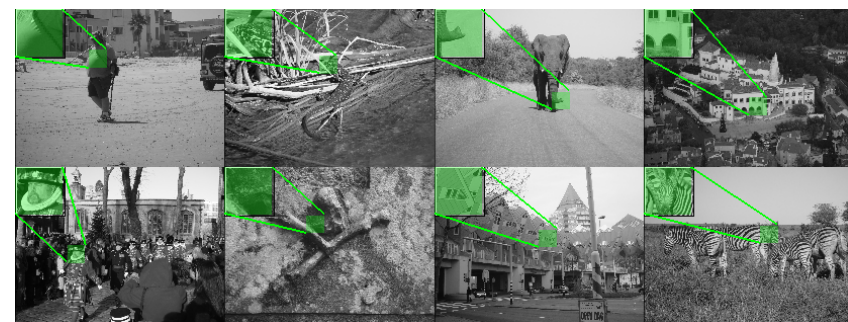

Fig. 15. The eight data sets used for testing the similarity metrics. The templates are the green shaded images in the upper left corner in each case, and the corresponding region in each image is indicated as a small green shaded region. The resolution is approximately three times higher than what was used in testing.

Bias was measured using a hierarchical grid search to locate the position of maximum MI, and measuring its Euclidean translation distance from the ground-truth in pixels. The hierarchical grid search proceeded as follows. A $31 \times 31$ grid of positions was selected around the ground truth. The initial spacing (in both dimensions) between the grid points was 0.2 pixels, i.e. the grid covered a $6 \times 6$ pixel region. The MI was measured at each grid point. The grid was re-centered on the grid-point with the maximum MI and the grid-spacing reduced by a factor of 1.5. This procedure was repeated seventeen times. Hence, the final MI maximum was found to within a precision of $\pm 10^{-4}$ pixels, which was the termination criterion for the Powell optimisation.

In addition to bias, local maxima exist, to which the optimisation algorithm can erroneously converge. This effect was measured by finding the mean distance between the biased global maximum and the end-points of multiple tests after termination of optimisation. Optimisation was initiated from $100\left(x_{1}, x_{2}\right)$ positions each on six circles with radii ranging from 0.5 to 4 pixels. The template was not rotated; here only translation was being optimised. The starting points were offset by the same amount from the global maximum for all the MI estimation methods. The distance to the biased global maximum, rather than the ground truth, was used to decouple the effects of bias and local maxima.

Performance was measured for five template sizes ranging from $9^{2}$ to $17^{2}$ pixels, with a constant $(16 \times 16)$ number of bins. Performance was also measured as the number of bins in the joint histogram varied exponentially from $16 \times 16$ to $256 \times 256$ bins, with a constant template size of $17^{2}$ pixels. In all 288000 convergence tests were performed: 6 MI methods $\times 8$ images $\times(5$ template sizes +5 intensities $) \times 6$ radii $\times$ 100 initialisations.

Bias for varying numbers of bins is given for non-lattice aligned and lattice-aligned templates in Fig. 16. Bias was low, if non-zero, for lattice-aligned templates, in Fig. 16a, since the template and reference intensities exactly matched when aligned. Lattice alignment is unlikely in practice; these results are included to highlight the importance of considering the non-lattice aligned case. Hence the convergence results in Fig. 17 are plotted for lattice-offset templates only.

For bias of non-lattice aligned images, in Fig. 16p, NP Windows is the best performer and unlike the other methods is almost unaffected by the number of bins in the joint histogram.
For other methods, performance generally decreases as the number of bins increases, due to the lack of sufficient samples. In Fig. 16a, IPZ bias decreases with no. bins. This anomaly occurs because IPZ blurs the histogram slightly when offset from an exact match, increasing MI; hence the bias away from lattice aligned solutions. The blurring occurs over the window width, i.e. 1-2 bins, hence the greater the no. bins the less the effect.

Similar conclusions may be drawn from the results in Fig. 17. The graphs depict the average error for all 8 images and 100 starting positions for each method and initial distance. The non-zero errors for certain initialisations indicate that not all the registrations may have converged to the true alignment but some may have.

For STD, PVE and IPZ at low initial offsets, good performance occurs over the range of numbers of bins because the initial position is within the mountain-of-convergence (MOC). Likewise, at distant initial offsets, performance is poor in all cases, because the initial positions are mostly outside of the MOC. In some cases PVE shows the best performance at the largest initial offsets, because of a larger MOC size arising from extended spatial support. However at intermediate distances, near the edge of the MOC, there is a range of performances, and the quality of the similarity measure has a large influence on convergence. Notably, the number of bins has almost no influence on NP Windows in terms of convergence.

In Fig. 18, the results for bias while varying template size are given for both lattice aligned and non-lattice aligned templates. The convergence results, when varying template size, are plotted in Fig. 19 for lattice-offset templates only. In Fig. 18 , the bias generally decreases as the template size (and number of available samples) increases, because the statistics represent the data better and tend to dominate over the local blurring effects of the kernel (if one is used). This is particularly evident for PVE which has the largest bias despite being the closest competitor of NP Windows in terms of convergence. The number of pixels has a negligible effect on the uniformly low bias for NP Windows since it makes maximal use of available information and additional pixels add mainly redundant data.

In Fig. 19, the point of inflection shifts rightwards as the template size increases, because additional data can increase the size of the MOC. In the authors' experience, the radius of the MOC is generally around $10 \%$ of the template size, but depends on the dominant structure of the image. At large initial offsets, an improvement in performance as template size increases is a benefit in convergence tests since the information provided by boundary pixels (at least those closest to the position of correct alignment) drives the optimisation algorithm towards the position of alignment. This information, provided by boundary pixels, only becomes redundant when the template is almost correctly aligned where all the pixels provide information useful to the registration operation. This improvement is clearly demonstrated for PVE (due to its large spatial support) and NP Windows (due to its effective use of all available subpixel information). Resolution Aware NP Windows shows the greatest improvement in convergence as 
(a)

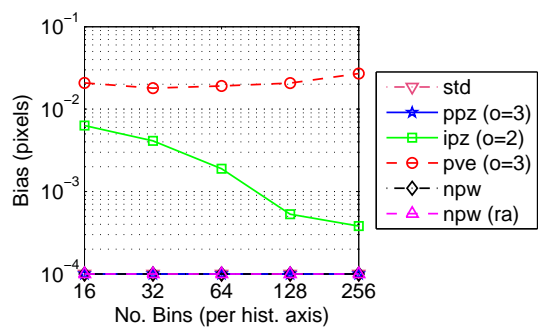

(b)

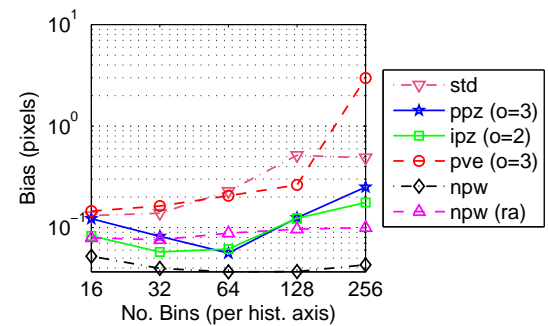

Fig. 16. Bias when varying the number of bins, for $(a)$ lattice aligned and $(b)$ non-lattice aligned templates. Both versions of NP Windows exhibit low bias for both templates - standard NP Windows performs the best. Moreover, both NP Windows techniques are unaffected by the number of bins.
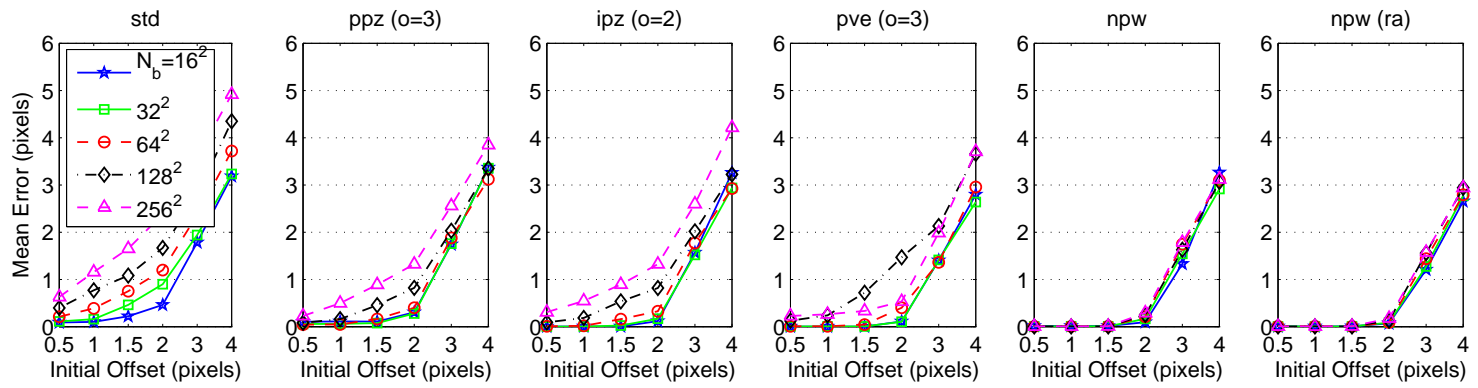

Fig. 17. Plots showing convergence (mean distance to biased ground truth for multiple tests) for various numbers of bins for lattice-offset templates. Both NP Windows techniques exhibit good convergence properties with respect to translation offsets. Note the relative "tightness" of the NP Windows plots indicating that performance is hardly affected by the number of bins.

(a)

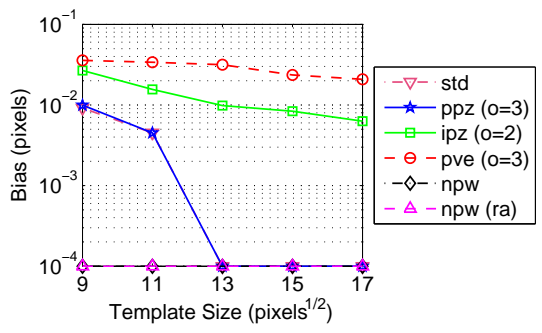

(b)

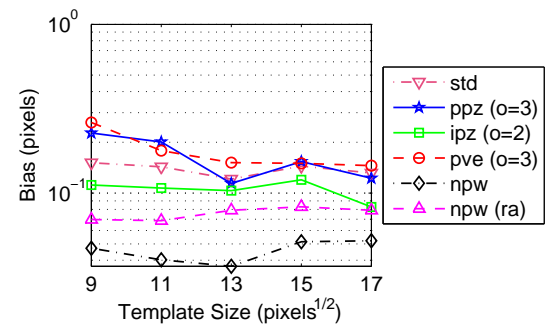

Fig. 18. Bias when varying template size, for $(a)$ lattice aligned and $(b)$ non-lattice aligned templates. NP Windows exhibits the least bias consistently across the various template sizes.
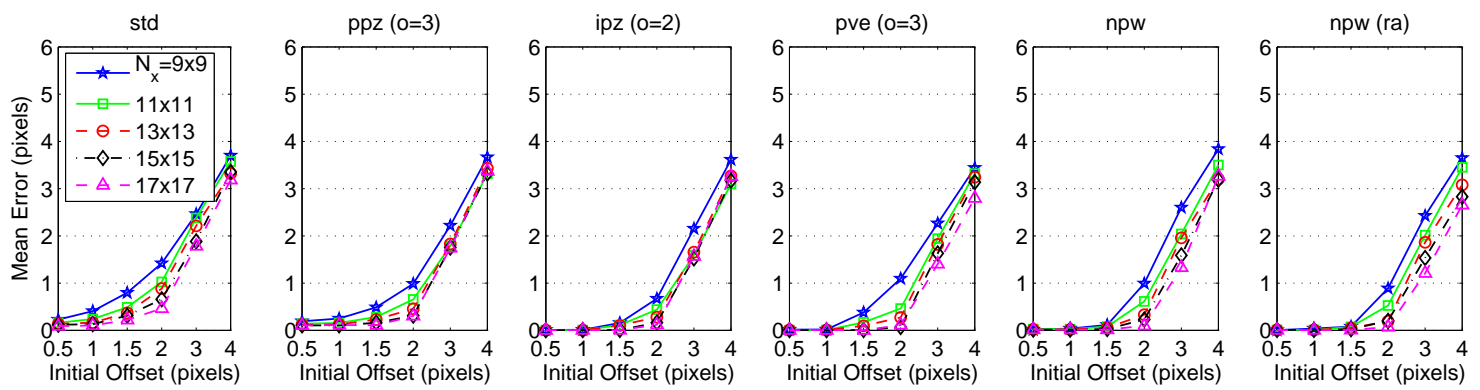

Fig. 19. Plots showing convergence for varying template sizes when using lattice-offset templates. NP Windows is virtually unaffected by the change in template size up to 1.5 pixels initial offset.

template size increases, because it models the intensity of the reference image better.

Figure 20 shows the variation in MI as a function of translation for the different methods as the number of bins is changed (in the upper plot) and as the template size is changed (in the lower plot). Unlike other methods, the NP Windows methods demonstrate virtual invariance to the bin and template size producing a useful MI function even with 256 bins. NP Windows shows a reduction in artifacts similar to PVE.

\section{B. Affine optimisation on large independent image pairs}

In this set of experiments, the convergence rate was measured for two images of $217 \times 181$ and $176 \times 154$ pixels, when optimising an affine transformation using the simplex [28] algorithm. Unlike in Section $\mathrm{V}-\mathrm{A}$, the template was not extracted from the reference image, but obtained independently. The images used were:

- Simulated PD and T2 weighted magnetic resonance images of a human brain [29]. 
(a)
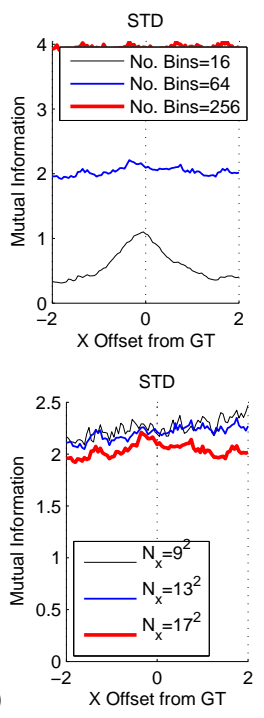

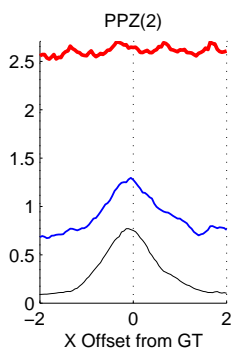

PPZ(2)

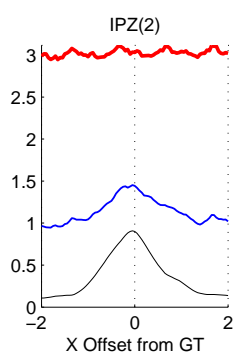

$\mathrm{IPZ}(2)$
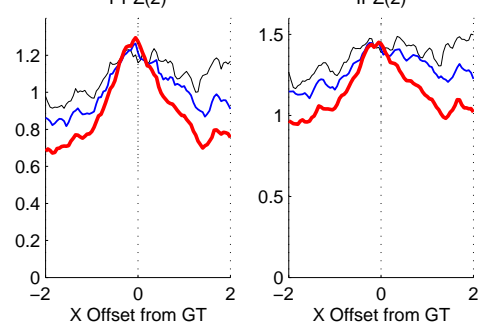

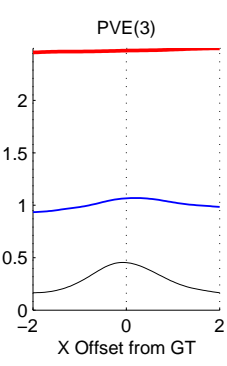

$\operatorname{PVE}(3)$

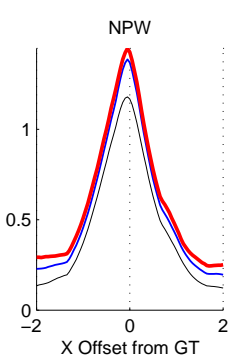

NPW
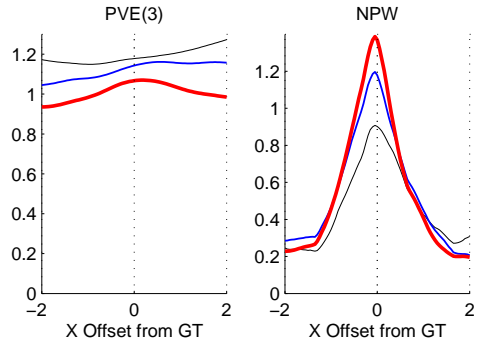

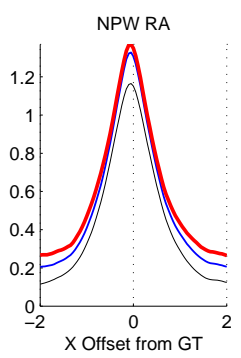

NPW RA

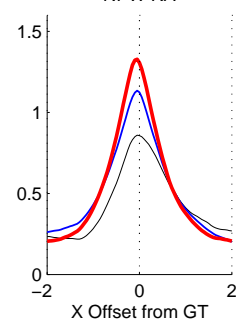

Fig. 20. Trace plots of various MI methods as the $x_{1}$ translation parameter is varied for the upper-left image in Fig. 15 In the upper plot, (a), the number of bins is varied for a fixed template size of $17 \times 17$. In the lower plot, (b), the template size is varied and $64 \times 64$ bins are used. The NP Windows traces show greater invariance to template size and number of bins, and they exhibit a greater range of values than other methods. Both of these properties are desirable for optimisation algorithms.

- Sagittal PET and CT images of a human thorax and head.

The brain images were simulated using the brainweb program described in [29] and hence had a known ground truth for registration. The thorax images were planes extracted from a hybrid PET/CT scan with a known ground-truth alignment. The images are shown in the leftmost column of Fig. 21. The second alignment was not perfectly accurate due to motion in the PET image, where acquisition time is much greater than that for the CT. However, the ground-truth was considered adequate for the purposes of this experiment, as is demonstrated by the function traces surrounding the ground-truth for translation and rotation parameters in Fig. 22 Rotation is shown instead of affine parameters as this is more intuitively understood. NP Windows had artifacts in both cases, although resolution awareness removed these.

In a similar manner to Section $\mathrm{V}$-A the $\left(x_{1}, x_{2}\right)$ parameters of three corner points of the template were offset from their ground truth position by a preset magnitude but at a random angle. The affine transformation, between each triplet of offset corner positions and the original corner positions, was used as an initial parameter value. Fifty such initialisations were generated for each offset. There are six affine parameters in all, consisting of four rotation components and two translation components, labelled: $\left(R_{11}, R_{12}, R_{21}, R_{22}, T_{x}, T_{y}\right)$.

Since the simplex method does not rely on the cost function being smooth, similar convergences were obtained for all the methods (including standard sampling, which would normally fail for other methods like Powell's method). At some points the error increases, even though MI increases, because the function surface topology contains multiple peaks and valleys with a gradient that does not always tend towards correct alignment. In general however, this is the exception. The convergence frequency to a final mean error of less 2 pixels at several initial offset magnitudes is plotted to the far right of each image. NP windows demonstrated consistently
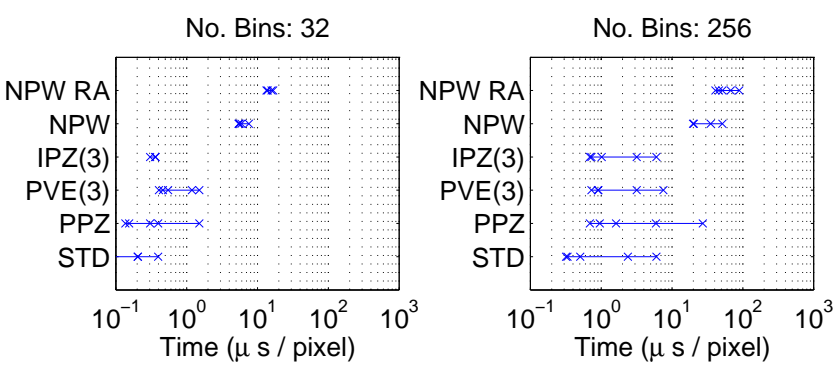

Fig. 23. Computational costs when evaluating MI using various methods.

better performance than the other MI estimation methods. The resolution aware approach gave the best overall performance because of its better modelling of the reference image at large differences in relative scale. Finally, the above tests were performed for the same images after being subsampled by four times in each dimension. The convergence frequency using the same criterion was measured, i.e. two pixels at the original resolution. PVE and NP Windows gave the best performance in this case.

\section{Computational Costs}

The computational cost was measured empirically for six MI estimation methods: Standard sampling, Post Parzen Windowing, third order PVE, third order IPZ, NP Windows and resolution aware NP Windows. Ten sets of measurements were taken for five template sizes, ranging from: $21 \times 21$ to $161 \times 161$ pixels in size. The mean time per template pixel for each set is shown in Fig. 23 The reason a range of computational costs occurs is that there is a certain computational overhead associated with each function evaluation. The cost asymptotes towards a minimum value as the number of template pixels increases, and as relative cost overhead diminishes.

Standard sampling is the cheapest method, as it performs 


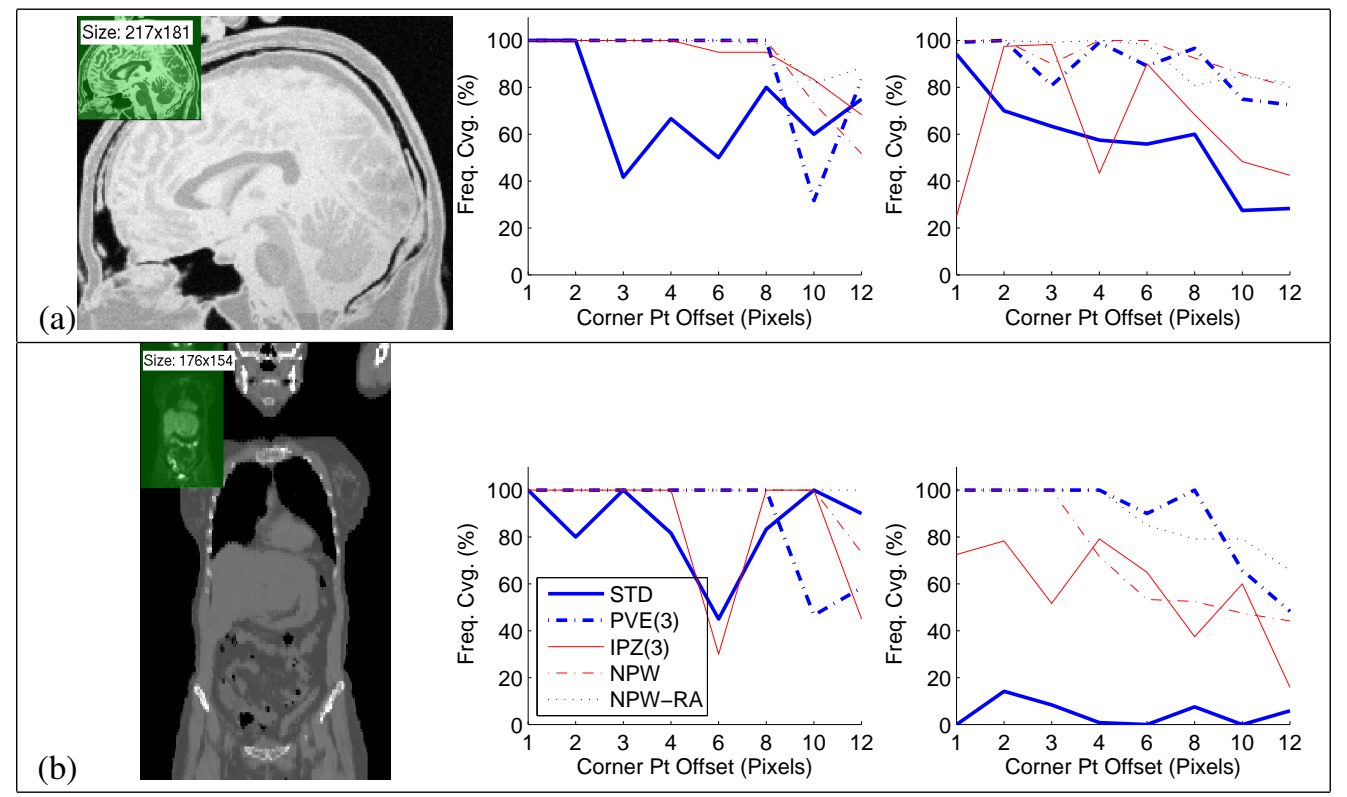

Fig. 21. Mean error during simplex optimisation of affine parameters for three image pairs: (a) a simulated PD and T2 weighted MR images of a brain, (b) sagittal PET and CT images obtained with a PET/CT machine. Column 1 shows the template and reference images. Column 2 shows the convergence frequency. Column 3 shows the convergence frequency for images after subsampling them by a factor of four.

(a)
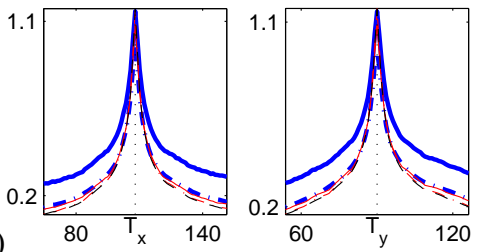

(c)
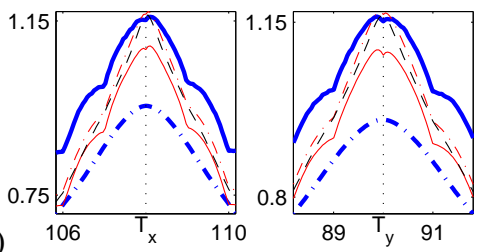
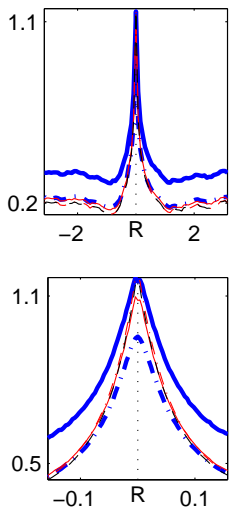

(b)
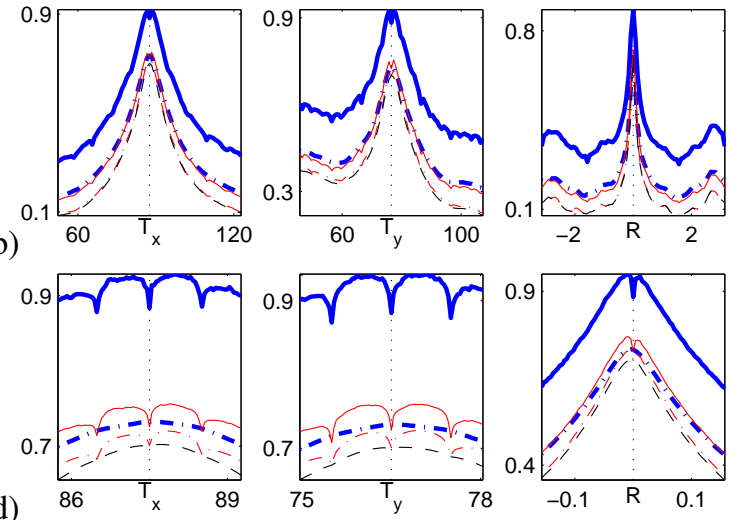

Fig. 22. Function traces for $\mathrm{x}$-translation, $\mathrm{y}$-translation and rotation parameters around the estimated ground-truth for the brain (column 1) and thorax (column 2) images, in Fig. 21 Top row shows a traces at macroscopic scale (40\% of template size). Bottom row shows traces at microscopic scale (4\% of template size). The legends are the same as those in Fig. 21

the fewest operations per sample. NP Windows is between 20 and 50 times as expensive as the other methods, because it renders two complete triangles for every pixel in the template, whereas the other methods write between one and sixteen bins in the histogram: a somewhat cheaper operation. The resolution aware version NP Windows is a further factor of three more expensive than NP Windows, as expected from the discussion in Section IV-C. Although NP Windows is an expensive operation, it is practical to use for registrations, where high accuracies are required. Moreover, due to its use of subpixel information, one might expect accurate results under substantial subsampling.

As a note on future work, to address the computational expense of NP Windows we have performed some preliminary experiments with hardware accelerated OpenGL. Since NP Windows for joint 2D images using half-bilinear interpolation is directly equivalent to additively rendering triangles onto the histogram, hardware accelerated graphics rendering may be used to substantially reduce the computational burden. For example, the time to render the $256 \times 256$ bin histograms for a pair of one Megapixel images was approximately $0.26 \mathrm{~s}$ for OpenGL NP Windows, versus $0.55 \mathrm{~ms}$ for standard sampling, 1.1s for third order PVE, 0.91s for third order IPZ and 22s for the software NP Windows implementation. Moreover, early experiments indicate that the number of bins in the histogram did not affect the speed of the algorithm. Compared to the other joint histogram estimation techniques NP Windows is almost trivial to implement in graphics hardware. However, we have found that the OpenGL rendered histogram is less accurate than its software equivalent because of approximations made by the graphics hardware. The approximations made are of the order of $5-15 \%$ and depend upon the number of bins. Therefore, we consider this to be preliminary work, reported here only to indicate a possible direction of future work; we aim to report further progress at a future date. 


\section{CONCLUSION}

In this paper, an NP Windows based method for obtaining the joint distribution of a pair of images was introduced. Unlike existing methods the proposed approach considers the signal as it varies between adjacent samples in a pair of images. NP Windows does not require arbitrary parameters like kernel size to be chosen, and it substantially reduces the error caused by only having a finite number of samples or bin-sizes.

Improvements to the NP Windows theory were proposed which used Green's Theorem to give a simpler implementation. NP Windows was extended to consider joint histograms of a pairs of images using bilinear interpolation. The crossterm in the bilinear equations resulted in a computationally complex and expensive algorithm. Half-bilinear interpolation was proposed to reduce the complexity, which resulted in an elegant method that used standard triangle rendering methods to generate a histogram.

The histograms obtained with NP Windows were used in registration applications to maximise the Mutual Information between a reference image and a template with respect to some warp parameters. The registration accuracy was compared to existing state of the art registration methods using MI. Performance was measured in terms of bias and convergence using a set of eight images at a range of initial starting points, when optimising for translation. In addition, the convergence rate and convergence frequency was measured when optimising an affine transformation between two pairs of large images.

NP Windows had less (translation) bias than any of the methods it was compared to (more than $40 \%$ less than its nearest competitor in some cases). The number of histogram bins did not significantly affect NP Windows, and the convergence rate steadily improved as the template size increased, implying that NP Windows makes maximal use of available data. NP Windows typically demonstrated the best convergence properties when testing registration for translation and affine parameters, although the large spatial support of third order PVE gave advantages when converging from initial positions far from the function maximum. Despite these results, NP Windows has not been shown to totally eliminate interpolation artifacts [14], only reduce them in some cases. Since artifacts stem from harmonics between the template and reference lattices [12], standard NP windows also has artifacts. Resolution awareness mitigates their effect. Resolution awareness further improved NP Windows performance, since it models the reference image better than other methods (for affine warps), especially when the scales of the two images differ substantially.

The test data and code used for this work are available on the authors website at www.robots.ox.ac.uk/ timork/npwindows.

NP Windows is a comparatively expensive histogram estimation technique, but is the method of choice when the MI value must be robust to number of bins and the number of samples is limited or unknown. The issue of computational cost may potentially be overcome by using graphics hardware to accelerate the approach. In domains where the bias (shift in true global maximum) must be kept to a minimum, NP
Windows is recommended due to its high accuracy compared to other methods. Possible applications could include small template matching, tracking, keypoint matching in large images and registration of heavily subsampled images.

There is a substantial amount of future work to be done with NP Windows. Extensions to deal with joint 3D data sets would be useful, with application to the registration of volumetric medical images. To achieve this, the cross terms can be eliminated in an approach similar to that presented in Section IV-A. The size of many medical images, such as PET or CT, are typically large and so either substantial subsampling will have to be performed or an OpenGL hardware implementation used. Results presented in this paper indicate that NP Windows should perform consistently under sub-sampling.

In addition, analytic first and second order derivatives of MI when using NP Windows would be useful. This would allow Newton-type optimisation methods to be used in registration applications, which would yield speed improvements. There will be some interesting associated challenges, because the derivative distributions are dependent on the image parameters, $x_{1}$ and $x_{2}$, in some cases making explicit numerical integration mandatory [30]. The Green's theorem approach discussed in Section III will be useful in dealing with this.

Non-linear transformations also need to be investigated. Two possible directions exist to solve this issue: using Green's Theorem to explicitly solve the transformation formula, or linearising the transformation locally. Finally, the NP Windows approach could be made more accurate by considering more optimal (and possibly non-invertible) interpolation models based on the known characteristics of the sensor being used to generate the image.

Although there is much future work, the wide applicability of NP Windows has been demonstrated. It has many useful properties, in particular its superior convergence and high accuracy (low bias). It allows MI values that are robust to limited numbers of samples, and are stable under varying numbers of bins, without requiring the pre-selection of a kernel.

\section{ACKNOWLEDGEMENTS}

Many thanks to the reviewers for their high quality reviews and valuable comments. Thank you also to $\mathrm{T}$ Wright for his help and advice. This work would not have been possible without an ORD scholarship and a grant from CVSSP. This work was also partially supported by the EPSRC project LILiR.

\section{REFERENCES}

[1] P. Viola and W. Wells, "Alignment by maximization of mutual information," in Proc. Int'l Conf. on Computer Vision, Boston, MA, USA, June 1995, pp. 16-23.

[2] A. Collignon, F. Maes, D. Delaere, D. Vandermeulen, P. Suetens, and G. Marchal, Information Processing in Medical Imaging. Kluwer Academic, 1995, ch. Automated Multi-modality image registration based on information theory, pp. 263-374.

[3] C. Studholme, D. Hill, and D. Hawkes, "Automated 3D registration of truncated MR and CT images of the head," in Proc. British Machine Vision Conference, September 1995, pp. 27-36.

[4] P. Viola and W. Wells, "Alignment by maximization of mutual information," Computer Vision, Int'l Journal of, vol. 24, no. 2, pp. 137-154, 1997. 
[5] F. Maes, D. Vandermeulen, and P. Seutens, "Comparative evaluation of multiresolution optimization strategies for multimodality image registration by maximization of mutual information," Medical Image Analysis, vol. 3, no. 4, pp. 272-286, April 1999.

[6] F. Maes, D. Vandermeulen, and P. Suetens, "Medical image registration using mutual information," Proc. of the IEEE, vol. 91, no. 10, pp. 1699$1722,2003$.

[7] E. Loutas, N. Nikolaidis, and I. Pitas, "A mutual information approach to articulated object tracking," in Circuits and Systems, Proc. of Int'l Symposium on, vol. 2, Bangkok, Thailand, May 2003, pp. 672-675.

[8] R. Moddemeijer, "On estimation of entropy and mutual information of continuous distributions," Signal Processing, vol. 16, pp. 233-248, 1989.

[9] E. Parzen, "On estimation of a probability density function and mode," Annals of Mathematical Statistics, vol. 33, no. 3, pp. 1065-1076, September 1962

[10] P. Thevenaz and M. Unser, "Optimization of mutual information for multi-resolution image registration," Image Processing, IEEE Trans. on, vol. 9, no. 12, pp. 2083-2099, December 2000.

[11] F. Maes, A. Collignon, D. Vandermeulen, G. Marchal, and P. Suetens, "Multimodality image registration by maximisation of mutual information," Medical Imaging, IEEE Trans. on, vol. 16, no. 2, pp. 187-198, April 1997.

[12] H. Chen and P. Varshney, "Mutual information-based CT-MR brain image registration using generalised partial volume joint histogram estimation," Medical Imaging, IEEE Trans. on, vol. 22, no. 9, pp. 11111119, September 2003.

[13] E. D'Agostino, F. Maes, D. Vandermeulen, and P. Suetens, "An information theoretic approach for non-rigid image registration using voxel class probabilities," Medical Image Analysis, vol. 10, pp. 413-430, 2006.

[14] J. Pluim, J. Maintz, and M. Viergever, "Interpolation artefacts in mutual information-based image registration," Computer Vision And Image Understanding, vol. 77, pp. 211-232, 2000.

[15] T. Kadir and M. Brady, "Estimating statistics in arbitrary regions of interest," in Proc. British Machine Vision Conf., W. Clocksin, A. Fitzgibbon, and P. Torr, Eds., vol. 2, Oxford, 2005, September 2005, pp. 589-598.

[16] _ "Non-parametric estimation of probability distributions from sampled signals," Robotics Research Lab, Oxford, UK, Tech. Rep., July 2005.

[17] I. Matthews, T. Ishikawa, and S. Baker, "The template update problem," in Proc. 14th British Machine Vision Conference, R. Harvey and A. Bangham, Eds., University of East Anglia, Norwich, UK, September 2003.

[18] T. Kaneko and O. Hori, "Template update criterion for template matching of image sequences," in Proc. Int'l Conf. on Pattern Recognition, C. Y. Suen, R. Kasturi, and R. Plamondon, Eds., vol. 2, Quebec, Canada, August 2002, pp. 1-5

[19] A. Rajwade, A. Banerjee, and A. Rangarajan, "A new method of probability density estimation with application to mutual information based image registration,", in Proc. of Computer Vision and Pattern Recognition, D. Huttenlocher and D. Forsyth, Eds., New York, USA, June 2006.

[20] T. Cover and J. Thomas, Elements of Information Theory. New York: Wiley \& Sons, 1991.

[21] C. Shannon, "A mathematical theory of communication," The Bell System Technical Journal, vol. 27, pp. 379-423, 623-656, July, October 1948.

[22] A. Papoulis and S. U. Pillai, Probability, Random Variables, and Stochastic Processes, 3rd ed. McGraw-Hill Inc., 1991, pp. 124-148.

[23] J. C. Morrison, "Fast anti-aliasing polygon scan conversion," in Graphics Gems, A. S. Glassner, Ed. Morgan Kaufmann, June 1990, pp. 63-83.

[24] P. S. Heckbert, "Generic convex polygon scan conversion and clipping," in Graphics Gems, A. S. Glassner, Ed. Morgan Kaufmann, June 1990, pp. 84-86.

[25] C. A. Shaffer and C. D. Feustel, "Exact computation of 2D intersections," in Graphics Gems III, D. Kirk, Ed. Morgan Kaufmann, June 1994, pp. 188-192.

[26] E. W. Weisstein, "Polygon: Area," http://mathworld.wolfram.com/PolygonArea.html, August 2007.

[27] W. H. Beyer, Ed., CRC Standard Mathemtical Tables, 28th ed. Boca Raton, FL:CRC Press, 1987, pp. 123-124.

[28] W. Press, S. Teukolsky, W. Vetterling, and B. Flannery, Numerical Recipes in C, 2nd ed. Cambridge Univ. Press, 1992.

[29] R.-S. Kwan, A. Evans, and G. Pike, "MRI simulation-based evaluation of image-processing and classification methods," Medical Imag ing, IEEE Trans. on, vol. 18, no. 11, pp. 1085-1097, Nov 1999, http://www.bic.mni.megill.ca/brainweb/.
[30] S. Baker and I. Matthews, "Lucas-kanade 20 years on: A unifying framework: Part 1," Robotics Institute, Carnegie Mellon University, Pittsburgh, PA, Tech. Rep. CMU-RI-TR-02-16, July 2002.

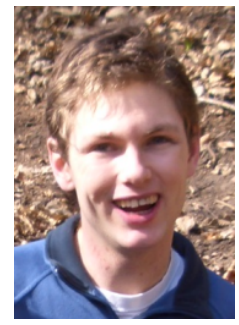

Nicholas Dowson received his BSc, summa cum laude, in Electical Engineering at the University of Witwaterand, Johannesburg in 1999. He took a somewhat circuitous route to reach the University of Surrey in 2003, which included starting a company and working on a process to automatically detect and ablate away errors in machine tools. He received a $\mathrm{PhD}$ from the University of Surrey in 2006. He now works as a research scientist at Siemens Molecular Imaging. His research interests are in tracking, registration and, more recently, in automatic object segmentation, localisation and classification. His work was supported by scholarships from the ORS and the Centre for Vision Speech and Signal Processing.

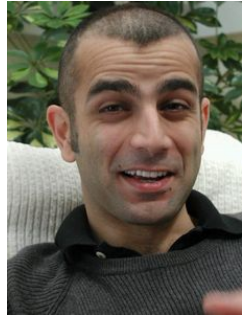

Timor Kadir graduated from the University of Surrey in 1996 with a MEng with Distinction in Electronic and Electrical Engineering and then worked at the Motorola European Research Laboratory investigating wireless image and video transmission systems and was active in ITU video transmission standardisation work groups. Following this he pursued a DPhil under the supervision of Professor Sir Michael Brady at the University of Oxford, completed in late 2001, where, amongst other things, he worked on salient feature detection and image segmentation and modelling. After two years as a post-doctoral researcher at Oxford working on techniques for affine invariant feature detection, distribution estimation and automated sign language interpretation he joined Siemens Molecular Imaging in 2004 (formerly Mirada Solutions) where he presently works as a Senior Scientist. His current research activities include medical image segmentation, anatomical labelling, image quantification and estimation of signal statistics. He has published a reasonable number of articles and has filed numerous patents.

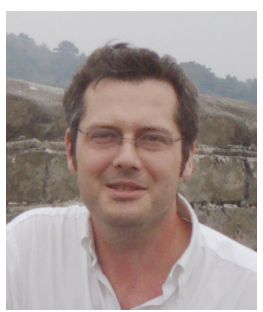

Richard Bowden received a BSc degree in Computer Science from the University of London in 1993, a MSc in 1995 from the University of Leeds and a PhD in Computer Vision from Brunel University in 1999. He is currently a Senior Lecturer at the University of Surrey, UK where he leads the Cognitive Vision Group within CVSSP. His research centers on the use of computer vision to locate, track and understand humans. His research into tracking and artificial life received worldwide media coverage, appeared at the British Science Museum and the Minnesota Science Museum. He has won a number of awards including paper prizes for his work on sign language recognition (undertaken as a visiting Research Fellow at the University of Oxford under subcontract from INRIA), as well as the Sullivan Doctoral Thesis Prize in 2000 for the best UK PhD thesis in vision. He was a member of the British Machine Vision Association (BMVA) executive committee and company director for 7 years. He is a London Technology Network Business Fellow, a member of the British Machine Vision Association, a Fellow of the Higher Education Academy and a Senior Member of the Institute of Electrical and Electronic Engineers. 